This item was submitted to Loughborough's Research Repository by the author.

Items in Figshare are protected by copyright, with all rights reserved, unless otherwise indicated.

\title{
LES of recirculation and vortex breakdown in swirling flames
}

PLEASE CITE THE PUBLISHED VERSION

PUBLISHER

(C) Taylor \& Francis

VERSION

AM (Accepted Manuscript)

LICENCE

CC BY-NC-ND 4.0

REPOSITORY RECORD

Malalasekera, W., K.K.J. Ranga-Dinesh, Salah S. Ibrahim, and Assaad R. Masri. 2019. "LES of Recirculation and Vortex Breakdown in Swirling Flames". figshare. https://hdl.handle.net/2134/5574. 
This item was submitted to Loughborough's Institutional Repository (https://dspace.lboro.ac.uk/) by the author and is made available under the following Creative Commons Licence conditions.

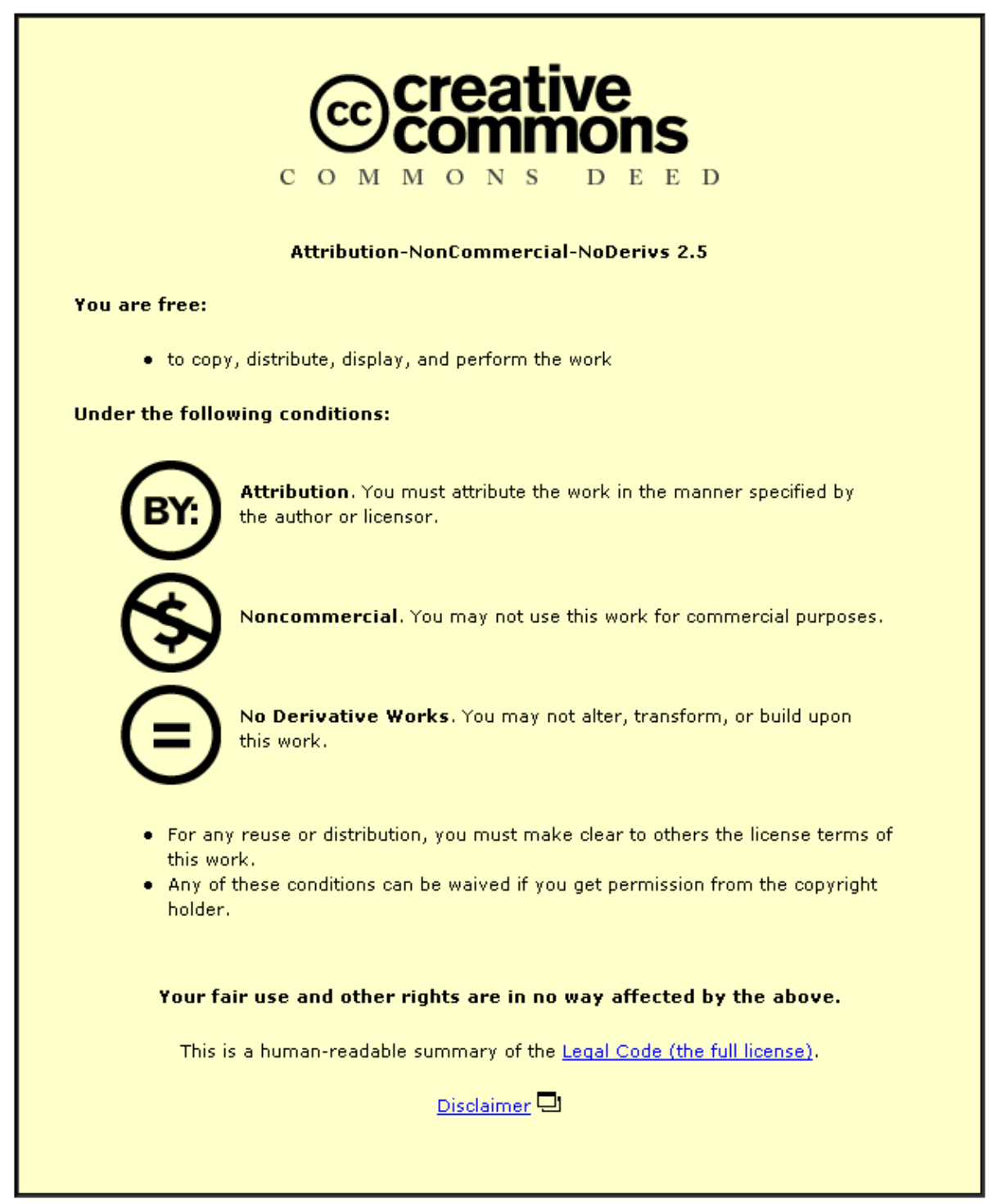

For the full text of this licence, please go to: http://creativecommons.org/licenses/by-nc-nd/2.5/ 
Citation:

Malalasekera, W., Ranga-Dinesh, K.K., Ibrahim, S. and A.R.Masri,

LES of Recirculation and Vortex Breakdown of Swirling Flames,

Combust. Sci. and Tech., 180: 809-832, 2008,

DOI : $10.1080 / 00102200801894018$.

\title{
LES OF RECIRCULATION AND VORTEX BREAKDOWN IN SWIRLING FLAMES
}

\author{
W.Malalasekera*, K.K.J.Ranga-Dinesh \\ Wolfson School of Mechanical and Manufacturing Engineering, Loughborough University,
} Loughborough, Leicestershire, LE11 3TU, UK.

S.S.Ibrahim

Department of Aeronautical and Automotive Engineering, Loughborough University Loughborough, Leicestershire, LE11 3TU, UK.

and

\section{A.R.Masri}

School of Aerospace Mechanical and Mechatronic Engineering, University of Sydney, Sydney, NSW 2006, Australia.

*_Corresponding author, e-mail:W.Malalasekera@lboro.ac.uk

October 2007

Paper presented at the $5^{\text {th }}$ Mediterranean Combustion Symposium, Monastir, Tunnia, 9-13 Septemeber, 2007. 


\title{
LES OF RECIRCULATION AND VORTEX BREAKDOWN IN SWIRLING FLAMES
}

\author{
W.Malalasekera $^{1}$, K.K.J.Ranga Dinesh ${ }^{1}$, S.S.Ibrahim ${ }^{2}$ and A.R.Masri ${ }^{3}$ \\ ${ }^{1}$ Wolfson School of Mechanical and Manufacturing Engineering, \\ ${ }^{2}$ Department of Aeronautical and Automotive Engineering, \\ Loughborough University, Loughborough, Leicestershire, LE11 3TU, UK. \\ ${ }^{3}$ School of Aerospace Mechanical and Mechatronic Engineering, University of Sydney, \\ Sydney, NSW 2006, Australia.
}

Keywords: Large eddy simulation (LES), Swirl, Combustion, Recirculation, Vortex breakdown (VB)

\begin{abstract}
In this study large eddy simulation (LES) technique has been applied to predict a selected swirling flame from the Sydney swirl burner experiments. The selected flame is known as the SM1 flame operated with fuel $\mathrm{CH}_{4}$ at a swirl number of 0.5 . In the numerical method used, the governing equations for continuity, momentum and mixture fraction are solved on a structured Cartesian grid. Smagorinsky eddy viscosity model with the localised dynamic procedure of Piomelli and Liu is used as the subgrid scale turbulence model. The conserved scalar mixture fraction based thermo-chemical variables are described using the steady laminar flamelet model. The GRI 2.11 is used as the chemical mechanism. The Favre filtered scalars are obtained from the presumed beta probability density function ( $\beta$-PDF) approach. The results show that with appropriate inflow and outflow boundary conditions LES successfully predicts the upstream recirculation zone generated by the bluff body and the downstream vortex breakdown zone induced by swirl with a high level of accuracy. Detailed comparison of LES results with experimental measurements show that the mean velocity field and their rms fluctuations are predicted very well. The predictions for the mean mixture fraction, subgrid variance and temperature are also reasonably successful at most axial locations. The study demonstrates that LES together with the laminar flamelet model in general provides a good technique for predicting the structure of turbulent swirling flames.
\end{abstract}




\section{INTRODUCTION}

Swirl stabilised turbulent flames are widely used in a range of practical combustion applications such as gas turbines, furnaces, power station combustors and boilers. Depending on the strength of swirl, a number of recirculation zones and central vortex breakdown regions can be seen in many swirl stabilised flames. Recirculation zones in swirl stabilised flames are effective in providing a source of well mixed combustion products and acts as storage of heat and chemically active species to sustain combustion and provide flame stabilization. Another type of a coherent structure referred to as precessing vortex core (PVC) which is an asymmetric three-dimensional time dependent flow structure is also present in some high swirl number flows. In general these features makes swirl flows and flames to exhibits highly three-dimensional, large scale turbulent structures with complex turbulent shear flow regions.

During the past four decades, a number of theoretical and experimental studies have been carried out to investigate the characteristics of swirling flames, which have mainly focused on instabilities and onset of vortex breakdown in combustion systems (see Syred and Beer, 1974, Gupta et al., 1984, Escudier, 1988, Lucca-Negro and O’Doherty, 2001). The complexity of the swirling flow behaviour depends on several key parameters such as the geometry of the working fluids and conditions that have been adopted in various research works to explore these phenomena (Escudier, 1988). Due to complex asymmetric and transient behaviour of swirling flames, a full theoretical or experimental description of the physical mechanism of recirculation and vortex breakdown has not been achieved.

Numerical calculation of swirl flows has also received considerable attention. However, the accurate prediction of recirculation and vortex breakdown, unsteady time dependent 
phenomena such as jet precession and asymmetric behaviour are computationally difficult problems to handle. Numerous researchers have applied different modelling approaches to predict swirling reacting and non-reacting flows in practical applications as well as in laboratory scale experiments. Majority of the attempts have used Reynolds averaged NavierStokes (RANS) equations accompanying different turbulence models to predict swirl flows. Sloan et al. (1986) and Weber et al. (1990) have reviewed much of these attempts. In generals RANS based models are primarily suitable to calculate stationary flows with non-gradient transport and they are not capable of capturing the unsteady nature of the large-scale flow structures found in swirl flows. Large eddy simulation (LES) technique on the other hand solves for large scale unsteady behaviour of turbulent flows therefore has been widely accepted as a promising numerical tool to accurately predict complex turbulent flows. Among others, the studies of Kim et al. (1999), Sankaran and Menon (2002), Di Mare et al. (2004), Wall and Moin (2005), Mahesh et al. (2005) have demonstrated the ability of LES to capture detailed flow field in swirling flow configurations.

In the computation of complex combusting flows the unsteady three-dimensional nature of LES has many advantages for turbulence modelling over classical Reynolds-averaged NavierStokes (RANS) approach. However in combustion LES, the chemical reaction usually occur well below to the resolution limit of the LES filter width and consequently modelling is required to predict the chemical state of the simulation. Combustion models which have been successfully used in the RANS context have been extended to LES to create sub-grid scale combustion models. For example, Cook and Riley (1994) applied equilibrium chemistry as a sub-grid model for the chemical reactions and Branley and Jones (2001) also applied a similar model to simulate a jet flame and obtained reasonably good predictions for the thermochemical variables. Forkel and Janicka (2000) have also demonstrated an efficient method for 
LES based on equilibrium chemistry. The steady flamelet modelling concept by Peters (1984) has been often used in combustion LES, because of its simplicity and ability to predict minor species. Venkatramanan and Pitsch (2005) and Kempf et al. (2006) have carried out combustion LES with the steady laminar flamelet model to simulate the Sydney bluff-body flames (Dally et al. 1998) and excellent comparisons with experimental measurements were obtained. However the steady flamelet assumption is not strictly valid for flows with slow chemical and physical process. The unsteady flamelet equations have to be used to account for such physical processes. Pitsch and Steiner (2000), for example, have demonstrated the Lagrangian unsteady flamelet model to simulate a piloted jet flame. A hybrid approach which employs LES and a particle based Lagrangian filtered-density-function approach by Raman et al. (2005), has also shown to give very good predictions in a bluff-body flame simulation. The conditional moment closure (CMC) model originally derived in the RANS context by Bilger (1993) has also been extended to LES. Kim and Pitsch (2005), Navarro-Martinez et al. (2005) have successfully demonstrated the conditional moment closure (CMC) model for LES. The flamelet/progress variable approach for LES proposed by Pierce and Moin (2004) has the potential to capture the local extinction, re-ignition and flame lift-off. Other approaches such as the linear eddy model developed by Mcmurtry et al. (1992) and the transported probability density function method originally introduced by Pope (1985) have also shown to be suitable for combustion LES.

Number of previous studies have demonstrated the application of combustion LES to swirling reacting and non-reacting flows. Wang et al. (2004) applied LES to study confined nonreacting turbulent swirling flows in a model dump combustor and successfully simulated the vortex breakdown, the circulation zones and anisotropic structures for all swirl numbers considered. Pierce and Moin (2004) have showed promising agreement between the LES 
results and the experimental measurements for low swirl number flames with the flamelet/progress variable approach.

The Sydney University swirl burner experimental series is one of the most useful experimental campaigns which have provided comprehensive data sets for reacting and nonreacting swirl flames. The swirl configuration used features a non-premixed flame stabilised by an upstream recirculation zone caused by a bluff body and second downstream recirculation zone induced by swirl, which greatly improve the mixing process. In certain cases of this flame series vortex breakdown has been observed. This flame series has also been the target flames for computations in the Proceeding of Turbulent non-premixed flames (TNF) group meetings (2006). The complexity of the flow conditions in these flames makes them ideal for the evaluation of turbulence chemistry interaction in combustion modelling.

In this paper, Large eddy simulation of a selected flame known as the SM1 flame from the Sydney swirl series is considered. The aim of this work is to investigate how LES technique employing moderate computing resources would perform in predicting key features of swirl flames. A number of other studies have attempted to model the Sydney swirling flame series with different modelling approaches. Masri et al. (2000) introducing this flame series have attempted to model a selected case using PDF-Monte Carlo approach using flamelet chemistry and showed good comparison for flow field parameters. No species or temperature comparison has been attempted in their study. El-Asrag and Menon (2005) studied the SM1 flame from this series using the linear eddy model for combustion LES and reported good agreement with data. More recently Stein and Kempf (2007) have attempted LES of one isothermal case (N29S054) and two combusting cases (SM1 and SMH1) using the laminar flamelet approach. Very good agreement for the flow properties of the non-reacting case and 
reasonable agreement for the mixture fraction and its variance in the SMH1 flame have been reported. Comparison of mean axial and tangential velocity predictions for the SM1 flame also showed good agreement. El-Asrag and Menon (2007), and James et al. (2007) have also carried out LES on selected cases and obtained encouraging results.

In a previous paper the present authors have shown LES predictions for different isothermal swirling flow fields of the Sydney swirl flame series with a good degree of success (Malalasekera et al. 2007). This paper is an extension of this earlier work where a reacting case is considered using combustion LES. The steady laminar flamelet model together with an assumed probability density function (PDF) approach is used for the combustion model. Here results obtained from combustion LES using moderate computer resources are compared with experimental data to asses the capability of LES.

The rest of the paper is structured as follows. Details of the burner configuration and the selected flame are discussed in the next section. In section 3, mathematical model for the governing equations and the combustion model details are presented and discussed. The numerical and computational detailed are presented in section 4, and in section 5 LES results obtained are compared with experimental measurements and discussed.

\section{SYDNEY SWIRL BURNER}

Figure 1 shows the Sydney swirl burner configuration, which is an extension of the wellcharacterised Sydney bluff body burner to swirling flames (Al-Abdeli and Masri, 2003). It has a 60mm diameter annulus for a primary swirling air stream surrounding the circular bluff body of diameter $\mathrm{D}=50 \mathrm{~mm}$. The central fuel jet is $3.6 \mathrm{~mm}$ in diameter. The burner is housed in a secondary co-flow wind tunnel with a square cross section of $130 \mathrm{~mm}$ sides. Swirl is 
introduced aerodynamically into the primary annulus air stream at a distance of $300 \mathrm{~mm}$ upstream of the burner exit plane and inclined 15 degrees upward to the horizontal plane. Swirl number can be varied by changing the relative magnitude of tangential and axial flow rates. The velocity measurements for mean velocity, rms fluctuations and Reynolds shear stresses were made at The Sydney University (Al-Abdeli and Masri, 2003) and compositional measurements in the combusting cases were made at Sandia National Laboratories (Masri et al., 2004).

The flow behaviour and flame characteristics were determined by four main parameters: the bulk axial velocity of fuel jet $\bar{U}_{j}$, the bulk axial and tangential velocity of primary air annuls $\bar{U}_{s}$ and $\bar{W}_{s}$, and the coflow velocity $\bar{U}_{e}$ of the wind tunnel. The swirl number is usually defined as the ratio between the axial flux of the swirl momentum to the axial flux of the axial momentum. In this experiment, a quantitative representation of the swirl intensity has been introduced by using the geometric swirl number $S_{g}$, which is expressed as the ratio of integrated (bulk) tangential to primary axial velocities $\bar{W}_{s} / \bar{U}_{s}$. This SM1 flame considered here used compressed natural gas (CNG) as the fuel, operated at a swirl number of 0.5 and used a fuel jet velocity of $32.7 \mathrm{~m} / \mathrm{s}$, which was $54 \%$ of the blow-off velocity. The Reynolds number was based on the fuel jet diameter of $3.6 \mathrm{~mm}$. Table 1 lists the details of the physical properties and characteristics of the flame SM1. In the experiments Laser Doppler velocimetry (LDV) technique has been used to measure the velocities. All the scalar measurements have been measured by using the Raman-Rayleigh technique.

\begin{tabular}{|c|c|c|c|c|c|c|c|}
\hline Case & Fuel & $U_{j} \mathrm{~m} / \mathrm{s}$ & $U_{s} \mathrm{~m} / \mathrm{s}$ & $W_{s} \mathrm{~m} / \mathrm{s}$ & $U_{e} \mathrm{~m} / \mathrm{s}$ & $S_{g}$ & $\mathrm{Re}_{s}$ \\
\hline SM1 & CNG & 32.7 & 38.2 & 19.1 & 20.0 & 0.5 & 75,900 \\
\hline
\end{tabular}

Table1. Details about the characteristics properties of flame SM1 


\subsection{Equations solved}

In LES the governing equations resolve the large scale features, which must be obtain by applying the filtering operator. The filtered field $\overline{f(x, t)}$ is determined by convolution with the filter function $G$.

$$
\bar{f}(x)=\int_{\Omega} f\left(x^{\prime}\right) G\left(x-x^{\prime}, \bar{\Delta}(x)\right) d x^{\prime}
$$

Where the integration is carried out over the entire flow domain $\Omega$ and $\bar{\Delta}$ is the filter width, which vary with the position. A number of filters are used in LES and a top hat filter having the filter-width $\bar{\Delta}_{j}$ set equal to the size $\Delta x_{j}$ of the local cell is used in the present work. In turbulent reacting flows large density variation occur and that is treated using Favre filtered variables. The transport equations for Favre filtered mass, momentum and mixture fraction are given by

$$
\begin{aligned}
& \frac{\partial \bar{\rho}}{\partial t}+\frac{\partial \bar{\rho} \tilde{u}_{j}}{\partial x_{j}}=0 \\
& \frac{\partial \bar{\rho} \tilde{u}_{i}}{\partial t}+\frac{\partial\left(\bar{\rho} \tilde{u}_{i} \tilde{u}_{j}\right)}{\partial x_{j}}=-\frac{\partial \bar{P}}{\partial x_{i}}+\frac{\partial}{\partial x_{j}}\left[\bar{\rho} v\left(\frac{\partial \tilde{u}_{i}}{\partial x_{j}}+\frac{\partial \tilde{u}_{j}}{\partial x_{i}}\right)-\frac{2}{3} \bar{\rho} \frac{\partial \tilde{u}_{k}}{\partial x_{k}}\right]+\frac{\partial \tau_{i j}}{\partial x_{j}}
\end{aligned}
$$

The transport equation for conserved scalar mixture fraction is written as

$$
\frac{\partial \tilde{\rho} \tilde{f}}{\partial t}+\frac{\partial}{\partial x_{j}}\left(\bar{\rho} \tilde{u}_{j} \tilde{f}\right)=\frac{\partial}{\partial x_{j}}\left[\bar{\rho}\left(\frac{v}{\sigma}+\frac{\sigma_{t}}{\sigma_{t}}\right) \frac{\partial \tilde{f}}{\partial x_{j}}\right]
$$

In the above equations $\rho$ is the density, $u_{i}$ is the velocity component in $x_{i}$ direction, $p$ is the pressure, $v$ is the kinematics viscosity, $f$ is the mixture fraction, $v_{t}$ is the turbulent viscosity, $\sigma$ is the laminar Schmidt number, $\sigma_{t}$ is the turbulent Schmidt number. An over-bar describes the application of the spatial filter while the tilde denotes Favre filtered quantities. 
The laminar Schmidt number was set to 0.7 and the turbulent Schmidt number for mixture fraction was set to 0.4 .

\subsection{Turbulence Model}

The subgrid contribution to the momentum flux is computed using Smagorinsky eddy viscosity model (Smagorinsky, 1963), which uses a model constant $C_{s}$, the filter width $\Delta$ and strain rate tensor $S_{i, j}$ according to equation (5):

$$
v_{t}=C_{s} \Delta^{2}\left|S_{i, j}\right|=C_{s} \Delta^{2}\left|\frac{1}{2}\left(\frac{\partial \tilde{u}_{i}}{\partial x_{j}}+\frac{\partial \tilde{u}_{j}}{\partial x_{i}}\right)\right|
$$

The model parameter $C_{s}$ is obtained through the localised dynamic procedure of Piomelli and Liu (1995).

\subsection{Combustion Model}

In LES, the chemical reactions occur mostly in the sub-grid scales and therefore consequent modelling is required for combustion chemistry. Here a presumed probability density function (PDF) of the mixture fraction is chosen as a means of modelling the sub-grid scale mixing. A $\beta$ function is used for the mixture fraction PDF. The functional dependence of the thermochemical variables is closed through the steady laminar flamelet approach. In this approach the variables, density, temperature and species concentrations only depend on Favre filtered mixture fraction, mixture fraction variance and scalar dissipation rate.

In the present selected case (SM1), there is no experimental evidence of significant local extinction. Hence a single flamelet with a strain rate of 500 /s has been used to calculate the characteristic flamelet profiles. This strain rate was chosen after comparing laminar flamelet profiles of density, temperature and species generated at different strain rates with 
experimental data and a rate of 500 /s seems to show a reasonably good agreement to be used as a single strain rate. The sub-grid scale variance of the mixture fraction is modelled assuming the gradient transport model proposed by Branly and Jones (2001). The flamelet calculations have been performed using the Flamemaster code (Pitsch, 1998) incorporating the GRI 2.11 mechanism for detailed chemistry (Bowman et al., 2006)

\section{NUMERICAL DESCRIPTION}

The program used to perform simulations is the PUFFIN code originally written by Kirkpatrick (2002) and later extended by Malalasekera et al. (2007). PUFFIN computes the temporal development of large-scale flow structures by solving the transport equations for the spatially filtered continuity, momentum and mixture fraction. The equations are discretised in space with the finite volume formulation (FVM) using Cartesian coordinates on a nonuniform staggered grid. Second order central differences (CDS) is used for the spatial discretisation of all terms in both the momentum equation and the pressure correction equation. This minimizes the projection error and ensures convergence in conjunction with an iterative solver. The diffusion terms of the scalar transport equation are also discretised using second order CDS. The convection term of the mixture fraction transport equation is discretised using the SHARP scheme (Leonard, 1987).

An iterative time advancement scheme is used for variable density calculation. First, the time derivative of the mixture fraction is approximated using the Crank-Nicolson scheme. The flamelet library yields the density and calculate filtered density field at the end of the time step. The new density at this time step is then used to advance the momentum equations. The momentum equations are integrated in time using a second order hybrid scheme. Advection terms are calculated explicitly using second order Adams-Bashforth while diffusion terms are calculated implicitly using second order Adams-Moulton to yield an approximate solution for 
the velocity field. Finally, mass conservation is enforced through a pressure correction step in which the approximate velocity field is projected onto a subspace of divergence free velocity field. The pressure correction method of Van Kan (1986) and Bell (1989) is the method used here. Typically 8-10 outer iterations of this procedure are required to obtain satisfactory convergence at each time step.

The time step is varied to ensure that the Courant number $C_{o}=\Delta t u_{i} / \Delta x_{i}$ remain approximately constant. Where $\Delta x_{i}$ is the cell width, $\Delta t$ is the time step and $u_{i}$ is the velocity components in the $x_{i}$ direction. The solution is advanced with a time stepping corresponding to a Courant number in the range of $C_{o}=0.3$ to 0.6 . The equations, discretised as described above, are solved using a linear equation solver. Bi-Conjugate Gradient Stabilized (BiCGStab) method with Modified Strongly Implicit (MSI) preconditioner are used to solve the system of algebraic equations resulting from the discretisation. The momentum residual error is typically of the order $10^{-5}$ per time step and the mass conservation error is of the order of $10^{-8}$.

The computational domain used dimensions $300 \times 300 \times 250 \mathrm{~mm}$ and employed a non-uniform Cartesian grid. Two different grid resolutions have been used to investigate the effect of the grid. Grid 1 consisted of $150 \times 150 \times 150$ cells (approximately 3.4 million) and Grid 2 consisted of $100 \times 100 \times 100$ cells ( 1 million) in $X, Y$ and $Z$ directions respectively. In the present case the mean axial velocity distribution for the fuel inlet and mean axial and swirling velocity distributions for air annulus are specified using power low profiles (Masri et al, 2000). A laminar velocity of $20 \mathrm{~m} / \mathrm{s}$ is used for the co-flow velocity. The fluctuations are generated from a Gaussian random number generator and added to mean velocity profiles 
such that the inflow has correct turbulence kinetic energy levels obtained from experimental data. A top hat profile is used as inflow condition for the mixture fraction. No-slip boundary condition is applied on the solid walls. At the outflow plane, a mass conserving convective outlet boundary condition is used for velocities and zero normal gradients is used for the mixture fraction.

The computations suggest that the statistical calculations can be started after 0.04s. This allows the flow field to fully develop and initial transients to exit the computational domain. The number of samples used for statistics is 1000 and corresponds to a sampling time of $0.02 \mathrm{~s}$ and the total time for the complete simulation is $0.06 \mathrm{~s}$. The length of the sampling interval used is sufficient to permit converged statistics.

\section{$4 \quad$ RESULTS AND DISCUSSION}

The Sydney swirl burner is designed to study reacting and non-reacting swirling flow structures for a range of swirl numbers and Reynolds numbers. The swirl induced recirculation and vortex breakdown leads to a very complex flow field, hence the accurate predictions of swirling flow field is important for the simulation of combustion, where an upstream bluff body stabilized recirculation zone and a downstream vortex breakdown zone can be seen certain cases. The structure of the swirling flow reveals the existence of rotating zones of gas within flames. Such rotating zones of gas lead to form the collar-like flow features (Al-Abdeli and Masri, 2003), where the flow dynamics are substantially different from those in the wider and adjoining flow.

The SM1 flame considered here is a short flame compared to other flames studied in the series. This flame operated with a jet velocity of $32.7 \mathrm{~m} / \mathrm{s}$ and a swirl number of 0.5 . The 
experiments showed upstream recirculation stagnating at about $43 \mathrm{~mm}$ downstream of the bluff body and a second down stream recirculation zone extending from about $65 \mathrm{~mm}$ to 110 $\mathrm{mm}$, centred around $70 \mathrm{~mm}$ in the axial direction. This recirculation zone results in from the vortex breakdown on the centre line of the geometry. A collar-like flow feature has been observed at about $60 \mathrm{~mm}$ from the burner surface. Oscillation of the central jet indicating the precession behaviour of this flame has also been reported (Al-Abdeli, 2003)

In our earlier work (Malalasekera et al. 2007), we have successfully predicted the nonreacting swirling flow fields and captured the occurrence of recirculation and vortex breakdown. The present work is an extension of the application to reacting turbulent nonpremixed swirling flames. The assessment of the capabilities of LES in predicting correct flow features such as recirculation zones and vortex breakdown in this combustion situation considered is aim of the work and the success is measured by comparing with detailed experimental data. Favre averaged velocities and scalars quantities are used in comparisons.

\subsection{Effect of the grid}

To gain an understanding of the effect of grid resolution on the LES results simulations were conducted using two different grids, a 3.4 million grid and a 1 million grid. The two grids are referred to as Grid 1 and Grid 2 respectively. Figure 2 shows the comparisons of the mean axial and swirl velocity between the measurements and LES results calculated using two grid resolutions. At upstream both grids yield similar results In further downstream, the Grid 1 results (3.4 million) give good predictions than Grid 2 results (1 million). Particularly, the mean axial velocity obtained with Grid 1 is much closer to the experimental measurements than Grid 2 in the outer shear layer of the downstream recirculation zone $(x / D=0.8,1.2)$.

Figure 3 shows the computed radial profiles of mean mixture fraction and its variance 
compared with experimental data for both grids. The Grid 1 results clearly show a good comparison with experimental data than Grid 2. The values of the centreline mean mixture fraction and the radial profiles of the mixture fraction variance are greatly improved with the fine grid simulation. However, overall results of these two grids as shown in the above comparisons are not vastly different from one another indicating that the fine grid is reasonably fine enough to produce good LES results and further refinement at an enormous cost is not required. In the following discussion Grid 1 (3.4 million cells) results are compared with experimental measurements.

\subsection{Flow Features}

Figure 4 (a) and (b) show snapshots of the filtered axial and swirl velocities. Figure 5 (a) and

(b) shows instantaneous mixture fraction and temperature respectively. These snapshots have been taken from animations, which provide an interesting insight into the complex transient turbulent swirling flow behaviour and combustion interactions. The animation of the filtered axial velocity contour plot shows the formation of the upstream and central recirculation zones where the axial velocity becomes negative and the dynamics of fuel jet break-up in the upstream recirculation zone can also be seen. The axial velocity animation show that the central recirculation zone formed by the vortex breakdown is very unstable in nature and appear to show a flapping behaviour. Figure 4(a) show the instantaneous nature of the negative velocity regions in the central zone and Figure 4(b) show the instantaneous swirling velocity where the swirl velocities are positive on one side and negative on the other side resulting in a collar-like flow. Mixture fraction in Figure 5(a) shows the breakup of the fuel jet and resulting mixture distribution within recirculation regions. Temperature animation in Figure 5(b) also show the dynamic nature of temperature distribution in the central vortex breakdown region. The stochiometric contour is also marked in Figure (b) to highlight the 
instantaneous high temperature regions and these regions indicate that the instantaneous temperature distribution is very much a dynamic feature and pockets of high temperature regions move about in axial and radial directions. Temperature animations indicate that the combustion products inside the recirculations zone continuously provide an ignition source, thereby stabilizing the flame. Zones and pockets of high temperature regions appear to be shed and consumed along the axial direction and these results in the flapping behaviour of the flame.

The contour plot for the mean axial velocity is shown in Figure 6. Recirculation zones and vortex breakdown bubble can be clearly identified in this mean contour pot. Here, LES appear to be very successful in reproducing all the flow features seen in the experiments. Stagnation region for the upstream recirculation zone where the mean axial velocity is zero is just above $40 \mathrm{~mm}$ which was observed in the experiments to be around $43 \mathrm{~mm}$. Contours shows that the stagnation region for the second vortex breakdown region is also predicted correctly which is about $70 \mathrm{~mm}$ from the burner surface.

\subsection{Velocity Field}

The success of the LES predictions is further demonstrated by the comparisons of the time averaged mean axial velocity, swirl velocity and rms values of axial and swirl velocities at different axial locations $x / D \in\{0.136,0.4,0.8,1.2,1.4,2.0$ and 2.5$\}$. Figure 7 shows the comparison of mean axial velocity with experimental data. The experimental data shows that there is a relatively short bluff body stabilized upstream recirculation zone towards the axial direction from the burner exit plane and a second central recirculation zone due to the occurrence of vortex breakdown (VB) around locations $x / D=1.4$ and 2.0. The negative values of the mean axial velocity at $x / D \in\{0.4,0.8\}$ and $x / D \in\{1 ., 2.0\}$ (Figure 7) indicate the 
flow reversal, which generate the upstream bluff body stabilized recirculation zone and the central VB zone respectively. It can be seen that LES predictions closely match the experimental data and correctly predict the upstream and central recirculation zones. These features can be seen in Figure 6 where the bubble shaped vortex breakdown zone is reproduced by the LES calculations. The downstream recirculation zone coincides with the highly rotating flow feature called collar-like flow as shown in Figure 6. The calculations have reproduced all peaks of the mean axial velocity well, which appears above the primary annulus (Figure 7). Overall axial mean velocity comparisons show very good agreement.

The comparison of the mean swirling velocity is shown in Figure 8. The comparisons between calculations and measurements are very good at most of the axial locations. The predictions have captured peaks appearing on the inner and outer shear layer of the upstream recirculation zone. However, at $x / D=0.136$ and 0.2 the swirl velocity is slightly over predicted above the bluff body face. This may be attributed to the shear layer instability and jet precession of the upstream recirculation zone which may not have been captured accurately by LES. At other down steam locations LES mean swirl velocity predictions closely follow the experimental measurements except at $x / D=2.0$ where the changes in swirl profile is not resolved that well. Overall agreement is, however, reasonably good for the mean swirling velocity.

Figure 9 shows comparison for the axial velocity fluctuations. The rms axial velocity fluctuations are found to be slightly under predicted at the first three axial locations and slightly over predicted at $x / D=1.2$ and 1.4 above the primary annulus. The overall agreement however is good for the rms axial velocity and its profiles are in reasonable agreement with measurements. Figure 10 shows the comparison of the swirl velocity 
fluctuations. The agreement between measurements and predictions is reasonable, but overprediction of the swirl velocity fluctuations can be seen near the central axis at locations $x / D=0.8$ and 1.2. Location $x / D=0.8$ corresponds to $40 \mathrm{~mm}$ axial distance which is the central region of the upstream recirculation zone. LES may not have captured very well the effects of interaction with the central precessing jet in this region. The location $x / D=1.2$ corresponds to $60 \mathrm{~mm}$ which is in the boundary of the two key features, upstream recirculation and downstream vortex breakdown. Again the effects of central jet precssion in this region may not have been very accurately captured by LES. It should be noted that the magnitude of both axial and swirl velocity fluctuations are small in comparison to their respective mean values and therefore discrepancies in these rms values are small in comparison.

Overall, the LES of SM1 yield a good qualitative and quantitative agreement with experimental observations for flow features, while some discrepancies are apparent. It should be noted that due to the coupling with density these discrepancies may have been resulted from certain deficiencies in combustion predictions which is discussed below.

\subsection{Scalar fields}

The instantaneous snapshot of the density and flame temperature is shown in Figure 5 (a) and (b). The calculations show two high temperature regions, one inside the upstream recirculation zone, the second located further downstream near to the centreline. Furthermore, the necking occurs around, $x=70 \mathrm{~mm}$ (downstream from the burner exit plane), which is linked to the collar-like flow feature and as a result, the visible flame width is reduced to about 25-30mm. It has been observed experimentally that, the flame SM1 is relatively shorter than other flames (Al-Abdeli and Masri, 2003). 
Figure 11 shows comparisons for the radial profiles of the mean mixture fraction at different downstream axial locations. It is evident that the radial spread of the mixture fraction is slightly under predicted in the regions between $r / R=0.4-0.8$ at $x / D \in\{0.2,0.4\}$. Despite this slight discrepancy, the agreement between calculations and measurements are good at other downstream axial locations except at $x / D=1.5$ where the mixture fraction at the centre line shows a notable over prediction. This is exactly the stagnation region of the central recirculation region where present LES may not have captured the correct mixture fraction distribution in this highly dynamic region. Figure 12 shows the computed profiles of mixture fraction variance with experimental data at different positions along the burner axis. The mixture fraction variance is also over-predicted at $x / D=1.5$. Overall predictions of mixture and its variance, however, shows reasonably good agreement all other locations.

The comparison of the predicted mean temperature field is shown in Figure 13. Given the complexity of the flow field, the comparison of the temperature field with experimental data is reasonable at most of the axial locations. It appears that the slightest under-prediction of the radial spread of the mixture fraction leads to a corresponding over-prediction of the temperature, which can be seen at locations $x / D \in\{0.2,0.4\}$. Although the mixture fractions and its variance predictions are reasonably good at locations $x / D=0.8$ and 1.2 the predicted peak temperature at the outer shear layer at these axial locations is somewhat over-predicted. The flame may be subjected high shear effects in this region and the use of a single flamelet to extract thermo-chemical properties may not be very accurate in this region. Furthermore, the steady flamelet assumption may not be perfectly valid in this region, which could have resulted in these discrepancies. As expected where the scalar predictions show notable discrepancy at $x / D=1.5$ the resulting temperature also show a corresponding under- 
prediction. Anther important aspect relevant to mixture fraction and its relationship to temperature is the stochiometric value of the mixture fraction. In this case the stochiometric mixture fraction value is 0.054 . Even a minor discrepancy around this value at locations where the mixture fraction should be 0.054 makes a noticeable difference in temperature. The over-prediction or under-prediction of temperature around peak temperature locations is tightly coupled to mixture fraction. Despite the discrepancies mentioned above the mean temperature profiles follow the correct experimental trends. The other studies which have used different approaches for combustion modelling to model this flame (James et al, 2007, El-Asrag and Menon, 2007) have reported similar success. It should also be noted that the radiation heat transfer is not included in the present simulations and account of radiative losses could results in reduced temperature profiles. The present LES show that there is scope for further improvements. We aim to improve the combustion modelling aspects and include radiative heat transfer calculations in our future work with combustion LES.

The comparison for the species concentration profiles are shown in Figures 14-16. The profiles for $\mathrm{H}_{2} \mathrm{O}$ are consistent with those with temperature with slightly similar peaks. Predicted $\mathrm{H}_{2} \mathrm{O}$ at locations $x / D=0.2$ and 0.4 show a very good match with experimental data while other profiles closely follow the experimental trends. The predictions of $\mathrm{CO}_{2}$ shown in Fig. 15 is slightly under-predicted at first two axial locations and considerably under-predicted at the location $x / D=1.5$. This is simple manifestation of the mixture fraction and temperature predictions discussed above for this location. Figure 16 shows CO predictions at axial positions showing similar trends as other species. In general, given the complexity of the flame and the flow field, present species predictions using the laminar flamlet model are reasonably good, however, there is scope for improvement in terms of 
combustion modelling as well as achieving good scalar predictions (mixture fraction and its variance).

The case considered here is a complex flow situation where there are upstream recirculation zones, a down stream vortex breakdown region, collar-like flow, and further experimental data shows that the central jet has a precession behavior. There are number of high shear layers and the interaction of flow, turbulence and chemistry is complex. The current LES attempt which used moderate resources has yielded reasonably good agreement with data and the study shows that the LES combustion procedure used is capable of predicting complex swirl flow situations. There may be regions in this flame where the steady laminar flamelet assumption may not be strictly valid and local extinction and re-ignition effects may be present which could have resulted in discrepancies where the present modeling approach does not address such issues. Overall the present LES attempt has shown successful results.

\section{CONCLUSION}

In this paper we have considered large eddy simulation of a turbulent swirling reacting flow test case from the Sydney swirl burner experimental series investigated by Masri and coworkers (Al-Abdeli and Masri, 2003; Masri et al., 2004). Here LES is performed for a swirling flame from the SM group known as the SM1 flame having a swirl number of 0.5. A Cartesian grid with 3.4 million nodes was used to perform the simulations. The steady laminar flamelet model that incorporates detailed chemical kinetics has been employed to obtain the thermo-chemical variables as a function of mixture fraction. The presumed beta probability density function approach has been used to model the sub-grid mixture fraction fluctuations. 
In the experiments a number of complex recirculation zones including a vortex breakdown zone has been observed. The predictions show that the LES has successfully captured the bluff body stabilized upstream recirculation zone and the downstream vortex breakdown (VB) region very well. The SM1 flame modelled also contain zones of gas, which rotate around the geometric centreline of the flow. These zones leads to the formation of the collar-like flow features downstream of the bluff body stabilized recirculation zone near the necking region of the flame. These features has been correctly predicted by the present simulation. Detailed comparison shows that the agreement between LES predictions and experimental data are good for mean and fluctuating velocity profiles, mean mixture fraction profiles and temperature. Given the complexity of the flow this is a good achievement and confirm the ability of LES to predict turbulence chemistry interactions in complex combusting flows. Some discrepancies between experimental data and predictions suggests that the steady laminar flamelet model may not be valid in some regions of swirling flames and further improvements of the combustion models will help to improve the temperature and species predictions.

\section{ACKNOWLEGEMENTS}

The authors would like to acknowledge the assistance received from Murthy Ravikanti VeeraVenkata of Loughborough University who helped to reproduce some of the figures.

\section{REFERENCES}

Al- Abdeli, Y.M. (2003), Experiments in turbulent swirling non-premixed flames and isothermal flows, Ph.D. Thesis, University of Sydney, Australia.

Al-Abdeli, Y.M, Masri, A.R. (2003), Stability Characteristics and flow fields of turbulent non-premixed swirling flames, Combust. Theo. Mode.., Vol. 7, pp. 731-766. 
Bell, J.B. Colella, P. and Glaz, H.M. (1989), A second order projection method for the incompressible Navier-Stokes equations, J. Comp. Phys., Vol. 85, pp. 257-283.

Bilger, R.W. (1993), Conditional moment closure for turbulent reacting flows, Phys. Fluids, Vol. A(5), pp. 436-444.

Bowman, C.T, Hanson, R. K, Davidson, D. F, Gardiner, W. C, Lissianki, V, Smith, G. P, Golden, D. M, Frenklach, M, Goldenberg, M. (2006), GRI 2.11, http://www.me.berkeley.edu/gri_mech

Branley, N and Jones,W.P. (2001), Large eddy simulation of a turbulent non-premixed flame, Combust. Flame, Vol.127, pp.1914-1934.

Cook, A.W, Riley, J.J. (1994), A subgrid model for equilibrium chemistry in turbulent flows, Phys. Fluids, Vol. 6(8), pp. 2868-2870.

Dally, B, Masri, A. R, Barlow, R, Fiechtner, G. (1998), Instantaneous and mean compositional structure of bluff body stabilised non-premixed flames, Combust. Flame, Vol. 114 , pp. 193-219.

Di Mare, Jones. W. Menzies. K. (2004), Large eddy simulation of a model gas turbine combustor, Combust. Flame, Vol.137(3), 278-294

Escudier, M. (1988), Vortex breakdown: observations and explanations, Prog. Aero. Sci., Vol. 25, pp. 189-229.

El-Asrag, H, Menon, S. (2005), Large eddy simulation of a swirling non-premixed flame, AIAA, Vol. 41, pp. 1-15.

El-Asrag, H, Menon, S. (2007), Large eddy simulation of bluff-body stabilised swirling nonpremixed flame, Proc. Combust. Inst., Vol. 31, pp. 1747-1754.

Forkel, H, Janicka, J. (2000), Large eddy simulation of a turbulent hydrogen diffusion flame, Flow Turbulence Combust, Vol.65, pp. 163-175.

Gupta, A.K., Lilly, D.G, Syred, N. (1984), Swirl Flows, Abacus Press, Kent, England. 
James, S, Zhu, J, Anand, M.S. (2007), Large eddy simulation of turbulent flames using filtered density function method, Proc. Combust. Inst. , Vol.31, pp. 1737-1745.

Kim, S.H, Pitsch, H. (2005), Conditional filtering method for large eddy simulation of turbulent non-premixed combustion, Phys. Fluids, Vol. 17(10), Art No. 105103.

Kim, W, Menon. S, Mongia, H. (1999), Large eddy simulation of a gas turbine combustor flow, Combust. Sci. Tech., 143, 25-63.

Kirkpatrick, M.P. (2002), Large eddy simulation code for industrial and environmental flows, PhD Thesis, Sch. Aero. Mech. and Mecha. Eng, University of Sydney, Australia.

Kirkpatrick, M.P, Armfield, S.W, Kent, J.H. (2003a), A representation of curved boundaries for the solutions of the Navier-Stokes equations on a staggered three dimensional Cartesian grid, J. Comput. Phy.,Vol 104, pp.1-36.

Kempf, A, Lindstedt, R.P, Janika, J. (2005), Large eddy simulation of bluff body stabilized nonpremixd flame, Combust. Flame., Article in press.

Leonard, B.P. (1987), SHARP simulation of discontinuities in highly convective steady flows, NASA Tech. Memo., Vol. 100240.

Lucca-Negro, O. and O’Doherty, T.O. (2001), Vortex breakdown: a review, Prog. Ener. Comb. Sci., Vol. 27, pp. 431-481.

Malalasekera, W, Ranga Dinesh, K.K.J, Ibrahim, S.S., Kirkpatrick, M.P. (2007). Large eddy simulation of isothermal turbulent swirling jets, Combut. Sci. Tech., Vol. 179, pp. 14811525.

Masri, A.R., Kalt, P.A.M., Barlow, R.S. (2004). The compositional structure of swirlstabilised turbulent nonpremixed flames, Combut. Flame, Vol. 137, pp. 1-37.

Masri, A.R., Pope, S.B. and Dally, B.B. (2000), Probability density function computation of a strongly swirling nonpremixed flame stabilised on a new burner, Proc. Combut. Inst., Vol. 28, pp. 123-131. 
Mahesh, K, Constantinescu, G, Apte, S, Iaccarineo, G, Ham, F, Moin, P. (2000), Large eddy simulation of turbulent flow in complex geometries, ASME J. App. Mech., Vol. 73,. 374381.

Mcmurtry, P.A, Menon, S, Kerstein, A. (1992), A linear eddy subgrid model for turbulent reacting flows: Application to hydrogen-air combustion, Proc. Combustion Inst. Vol.25, pp. 271-278.

Navarro-Martinez, S, Kronenburg, A, Di Mare, F. (2005), Conditional moment closure for large eddy simulations, Flow Turb. Combust., Vol. 75, pp. 245-274.

Peters, N. (1984), Laminar diffusion flamelet models in non-premixed turbulent combustion, Prog. Energy Combust. Sci., Vol. 10 , pp. 319-339.

Pierce, C.D. and Moin, P. (2004), Progress variable approach for LES of non-premixed turbulent combustion, J. Fluid Mech., Vol 504, pp. 73-97.

Piomelli, U. and Liu, J. (1995), Large eddy simulation of channel flows using a localized dynamic model, Phy. Fluids, Vol. 7, pp. 839-848.

Pitsch, H. (1998), A C++ computer program for 0-D and 1-D laminar flame calculations, $R W T H$, Aachen.

Pitsch, H, Steiner, H. (2000), Large eddy simulation of turbulent methane-air diffusion flame, Phy. Fluids, Vol. 12(10), pp. 2541-2554.

Pope, S.B. (1985), PDF methods for turbulent reacting flows, Prog. Energy Combust. Sci. , Vol. 11, pp. 119-145.

Proceedings of the $8^{\text {th }}$ International workshop on Turbulent Non-premixed Flames (TNF). (2006), Heidelberg, Germany, pp.11-75.

Raman, V, Pitsch, H, Fox, R.O. (2005), Hybrid Large eddy simulation/Lagrangian filtered density function approach for simulating turbulent combustion, Combust. Flame, Vol. 143, pp. 63-78.

Sankaran, V, Menon, S. (2002), LES of spray combustion in swirling flows, J. Turbulence, Vol.3, pp.11-23. 
Smagorinsky, J. (1963), General circulation experiments with the primitive equations”, $M$. Weather Review, Vol.91, pp.99-164

Sloan, D.G., Smith, P.J. and Smoot, L.D. (1986). Modelling of Swirl in Turbulent Flow Systems, Prog. Energy Combust Sci., Vol. 12, pp. 163-250.

Stein, O, Kempf, A. (2007), LES of Sydney swirling flame series: A study of vortex breakdown in isothermal and reacting flows, Proc. Combust. Inst. Vol. 31. pp. 17551763.

Syred, N and Beer, J.M. (1974), Combustion in Swirling Flows: A Review, Combust. Flame, 23, pp. 143-201.

Van Kan, J. (1986). A second order accurate pressure correction scheme for viscous incompressible flow, SIAM J Sci. Stat Comput., Vol. 7, pp. 870-891.

Venkatramanan, R, Pitsch, H. (2005), Large eddy simulation of bluff body stabilized nonpremixed flame using a recursive filter refinement procedure, Combust. Flame, Vol.142, pp.329-347.

Wall, C.T and Moin, P. (2005), Numerical methods for Large eddy simulation of acoustic combustion instabilities, Tech. Rep. TF.91. Stanford University, CA.

Weber, R, Visser, B.M, Boysan, F. (1990), Assessment of turbulent modelling for engineering prediction of swirling vortices in the near burner zone, Int. J. Heat and Fluid Flow, Vol.11, pp.225-238.

Wang.P and Bai, X,S, Wessman, M and Klingmann, J. (2004), Large eddy simulation and experimental studies of a confined turbulent swirling flow, Phy. Fluids, Vol.16, pp.33063324. 


\section{FIGURE CAPTIONS}

Figure 1: Schematic drawing of the Sydney swirl burner (adapted from Al-Abdeli and Masri, 2003).

Figure 2: LES predicted time averaged mean axial and swirl velocities using different grid resolutions. Dotted lines represent the Grid 1 results (finer grid), dashed lines represent the Grid 2 results (coarser), and symbols represent experimental measurements.

Figure 3: LES predicted mean mixture fraction and its variance using different grid resolutions. Dotted lines represent the Grid 1 results (finer grid), dashed lines represent the Grid 2 results (coarser), and symbols represent experimental measurements.

Figure 4: Snapshot of filtered axial (a) and swirl velocity (b).

Figure 5: Snapshot of filtered mixture fraction (a) and temperature (b).

Figure 6: Contour plot of mean axial velocity obtained from LES calculation.

Figure 7: Comparison of mean axial velocity. Lines represent LES results, and symbols represent experimental measurements.

Figure 8: Comparison of mean swirling velocity. Lines represent LES results, and symbols represent experimental measurements.

Figure 9: Comparison of rms of axial velocity fluctuations. Lines represent LES results, and symbols represent experimental measurements.

Figure 10: Comparison of rms swirling velocity. Lines represent LES results, and symbols represent experimental measurements.

Figure 11: Comparison of mean mixture fraction. Lines represent LES results, and symbols represent experimental measurements.

Figure 12: Comparison of mixture fraction variance. Lines represent LES results, and symbols represent experimental measurements.

Figure 13: Comparison of mean temperature. Lines represent LES results, and symbols represent experimental measurements.

Figure 14: Comparison of mass fraction of $\mathrm{H}_{2} \mathrm{O}$. Lines represent LES results, and symbols represent experimental measurements.

Figure 15: Comparison of mass fraction of $\mathrm{CO}_{2}$. Lines represent LES results, and symbols represent experimental measurements.

Figure 16: Comparison of mass fraction of $C O$. Lines represent LES results, and symbols represent experimental measurements. 


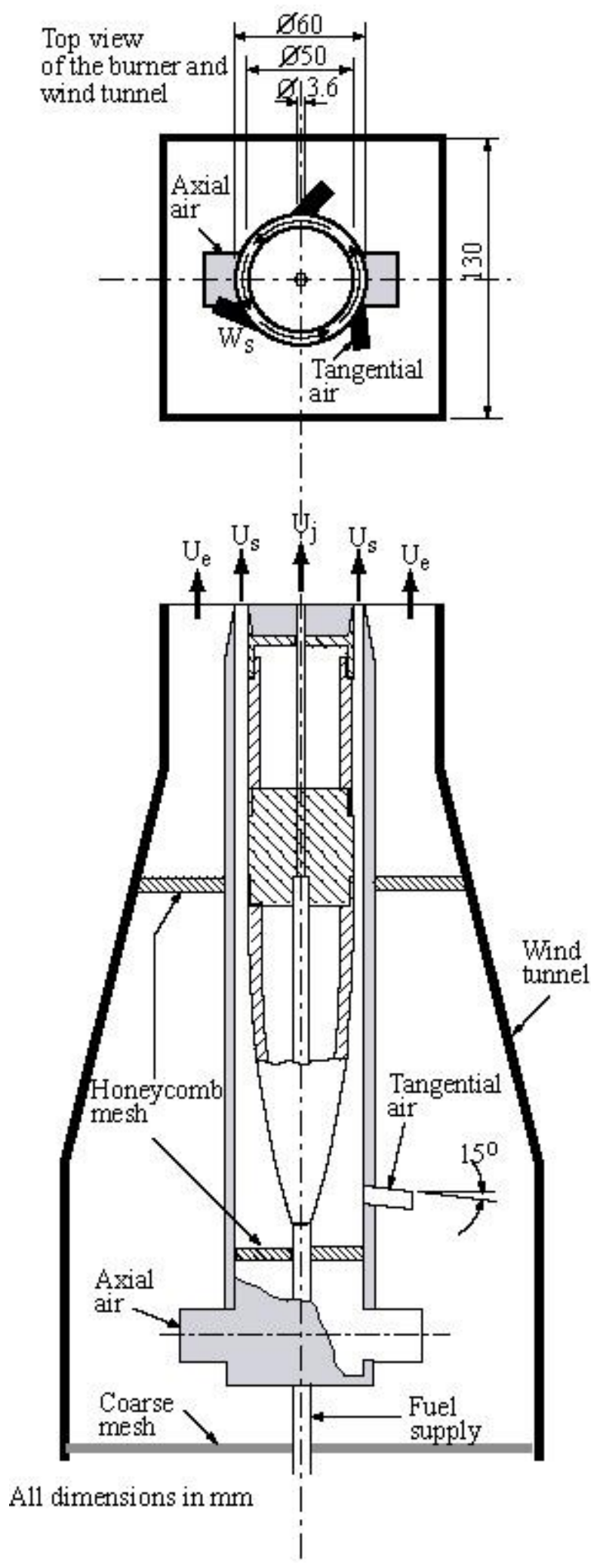

Figure 1: Schematic drawing of the Sydney swirl burner (adapted from Al-Abdeli and Masri, 2003). 

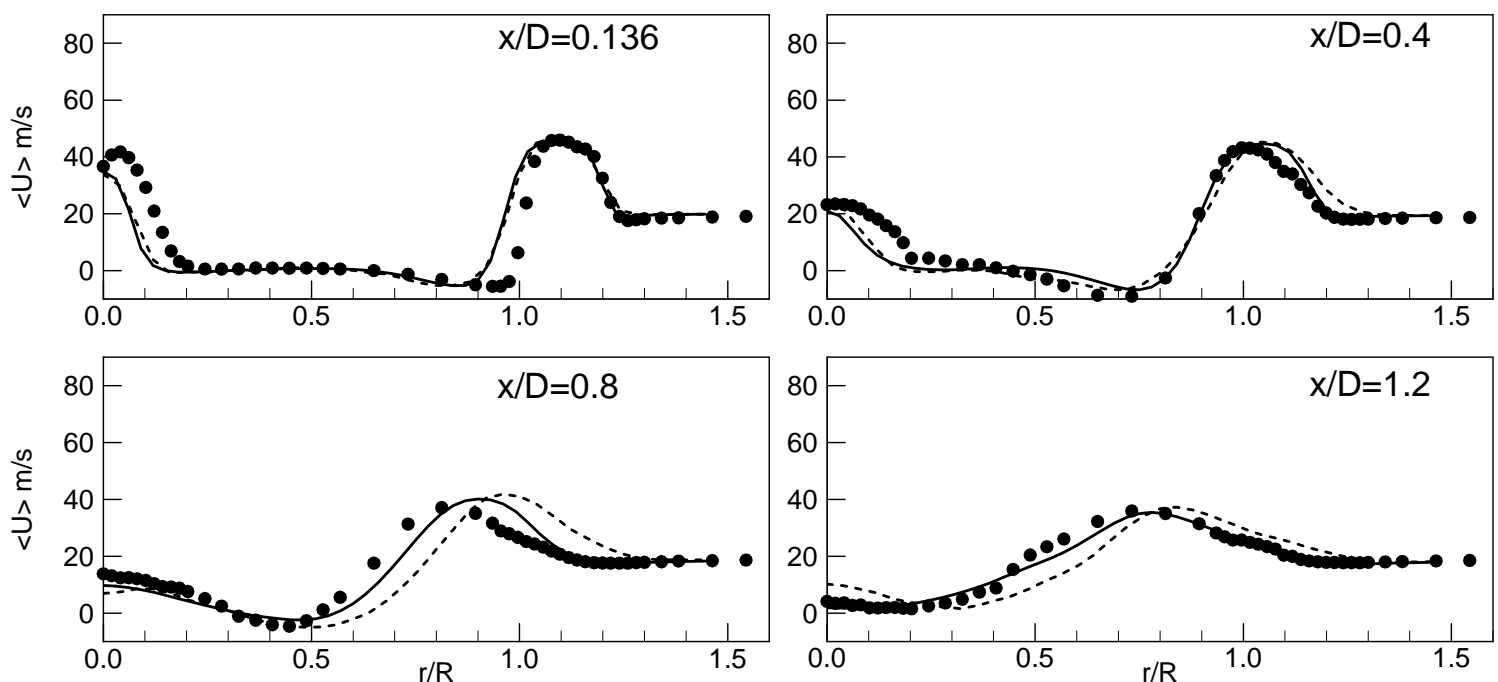

(a) Mean axial velocity
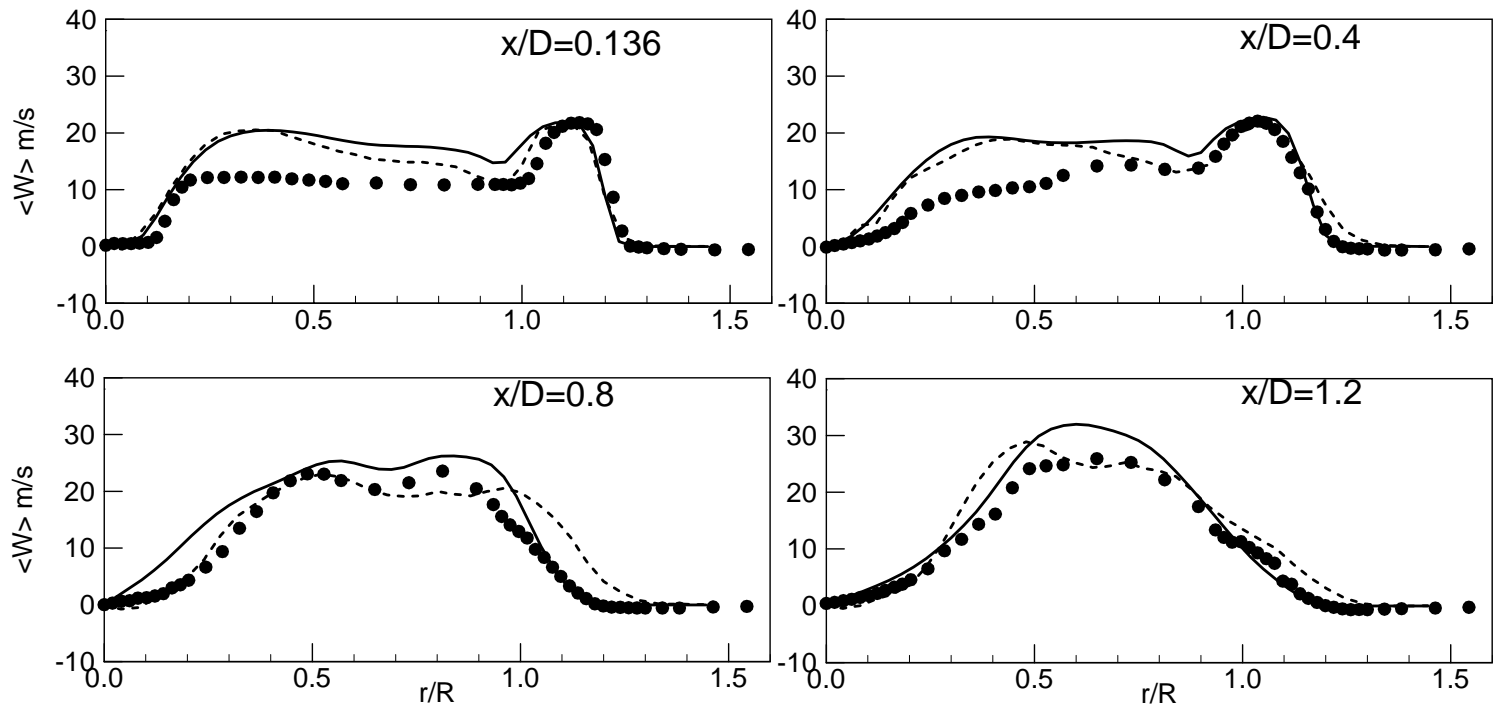

(b) Mean radial velocity

Figure 2: LES predicted time averaged (a) mean axial and (b) mean swirl velocity at different axial locations using different grid resolutions. Solid lines represent the Grid 1 results (3.4 million grid points), dashed lines represent the Grid 2 results (1 million grid points), and symbols represent experimental measurements. 

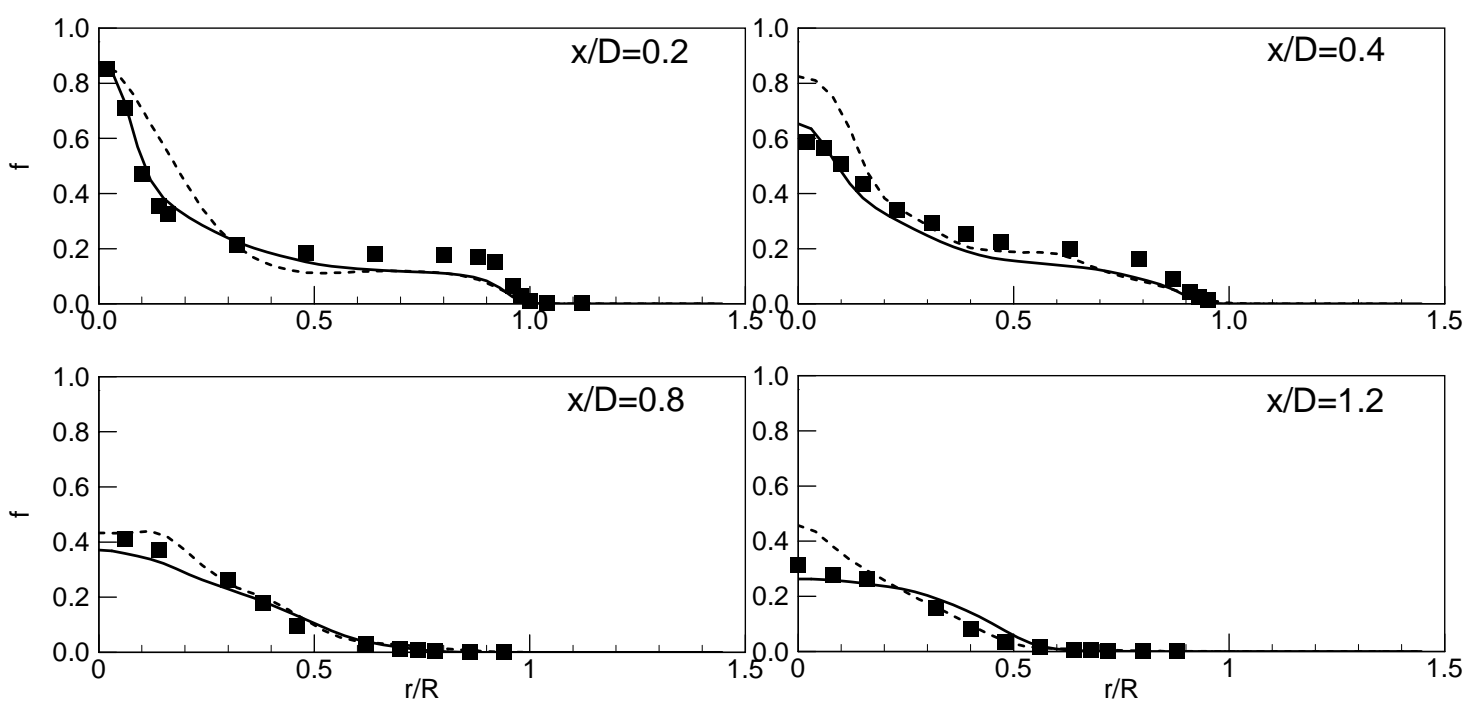

(a) Mixture fraction
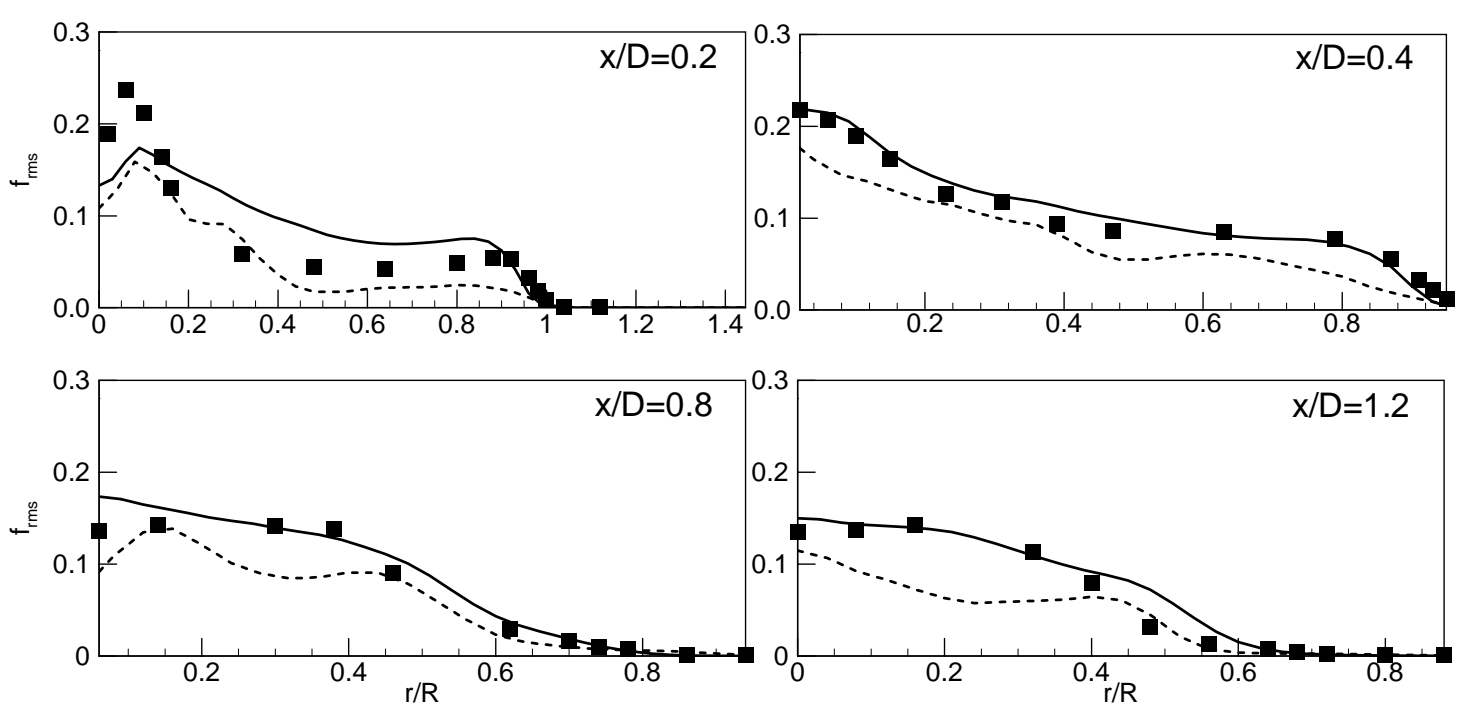

(b) Mixture fraction variance

Figure 3: LES predicted mean mixture fraction and its variance at different axial locations using different grid resolutions. Solid lines represent the Grid 1 results (3.4 million grid points), dashed lines represent the Grid 2 results (1 million grid points), and symbols represent experimental measurements. 


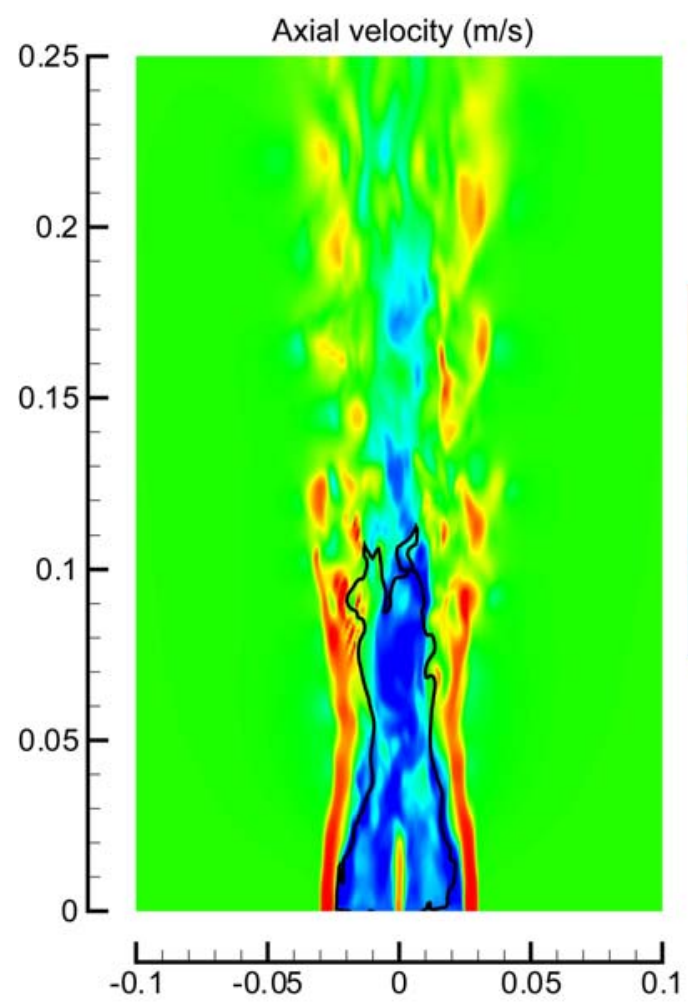

(a)

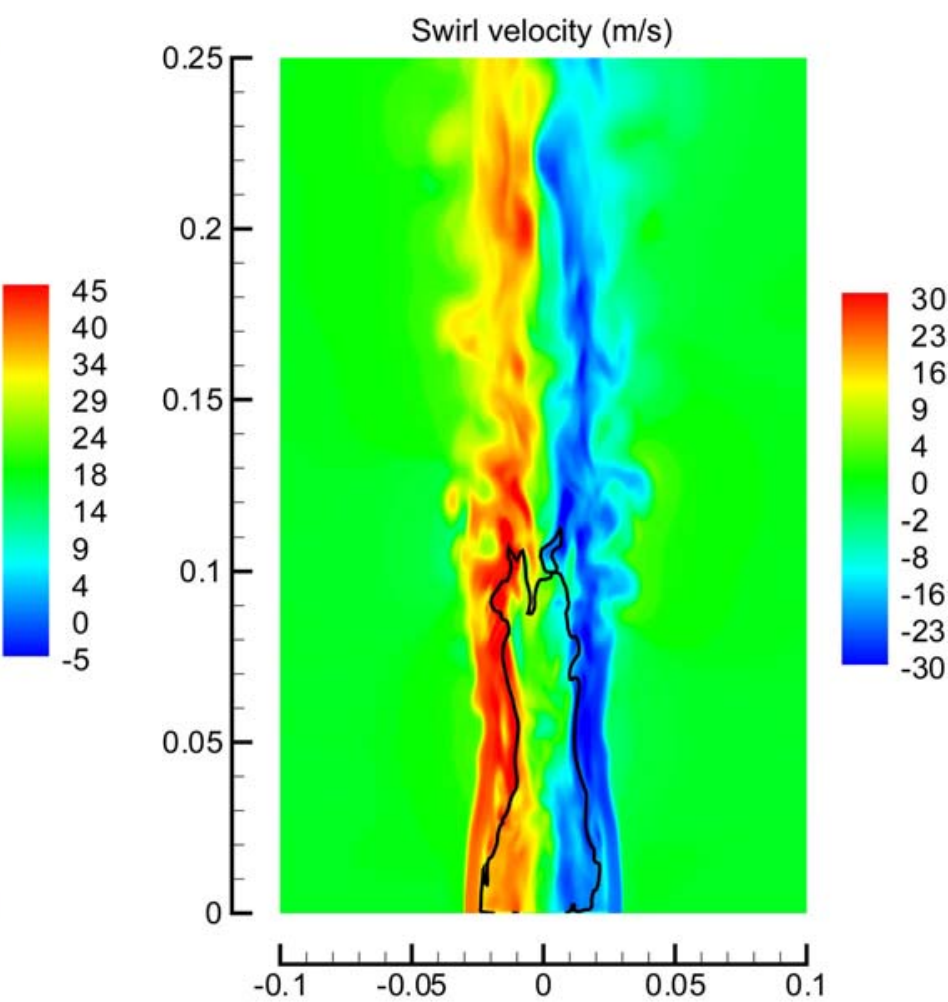

(b)

Figure 4: Snapshots of filtered axial and swirl velocity.

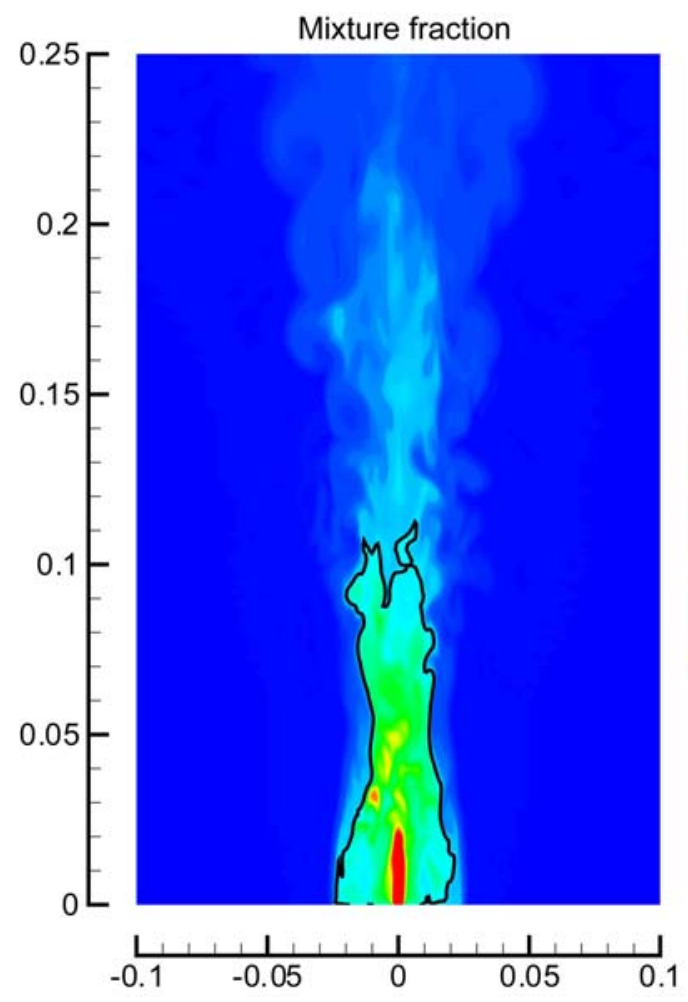

(a)

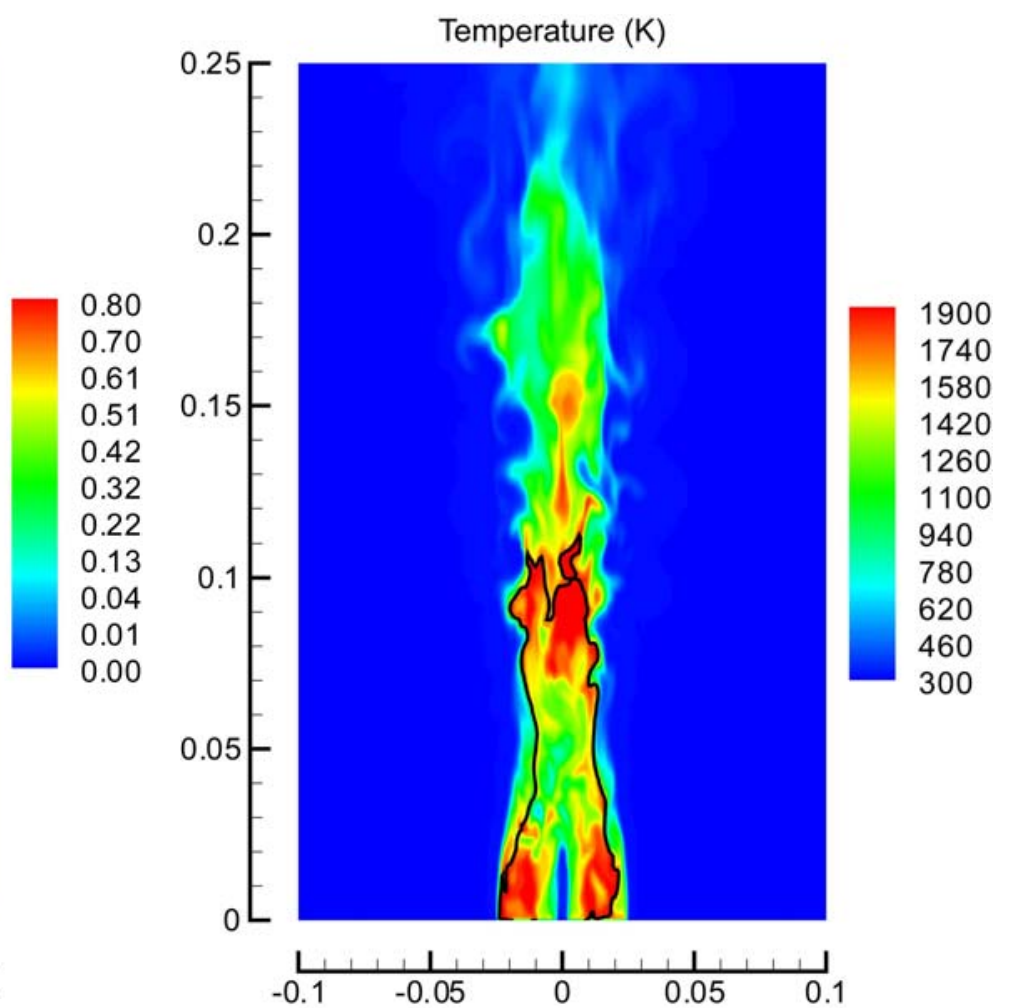

(b)

Figure 5: Snapshots of filtered mixture fraction and temperature. 


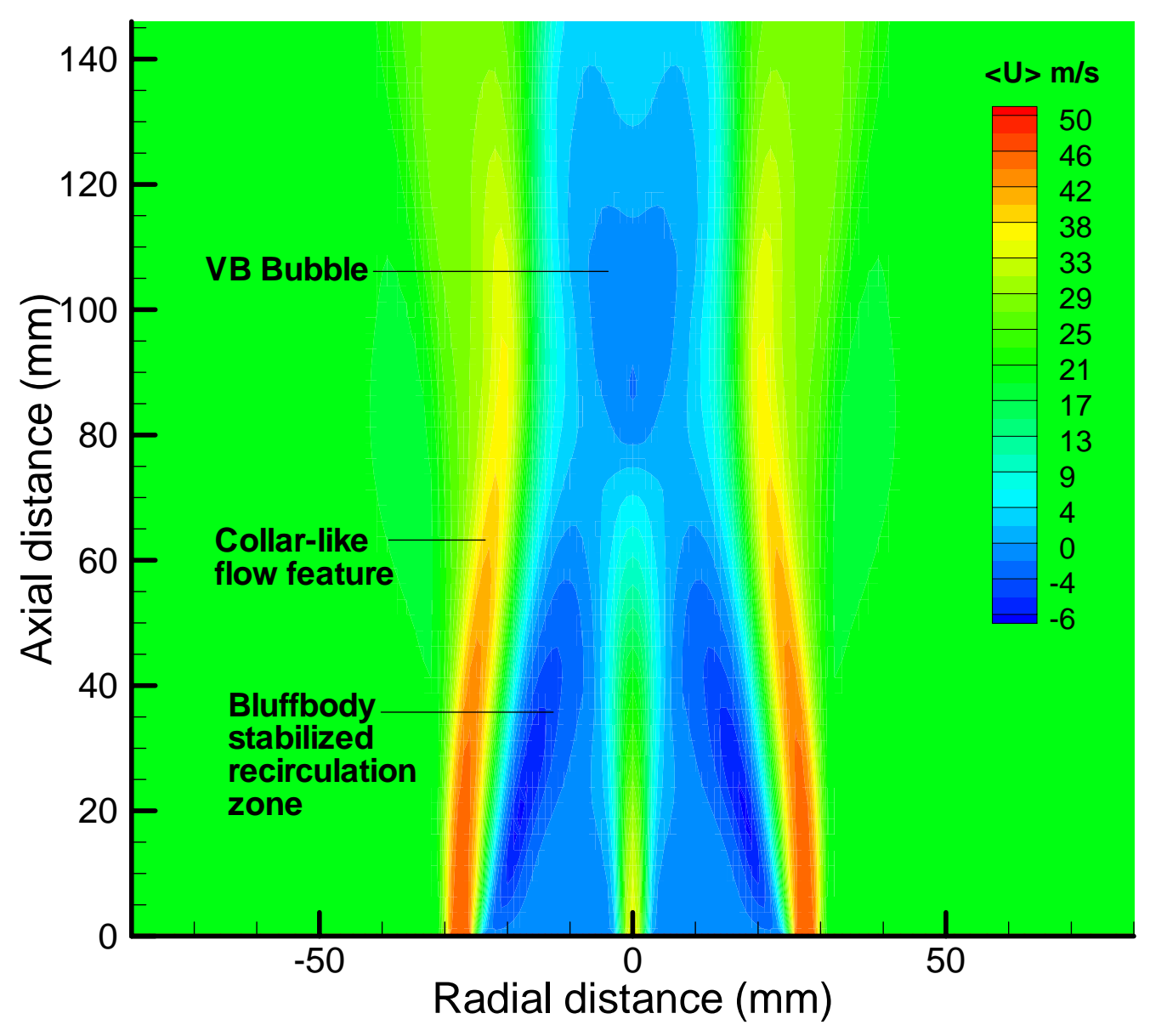

Figure 6: Contour plot of mean axial velocity obtained from LES calculation. 

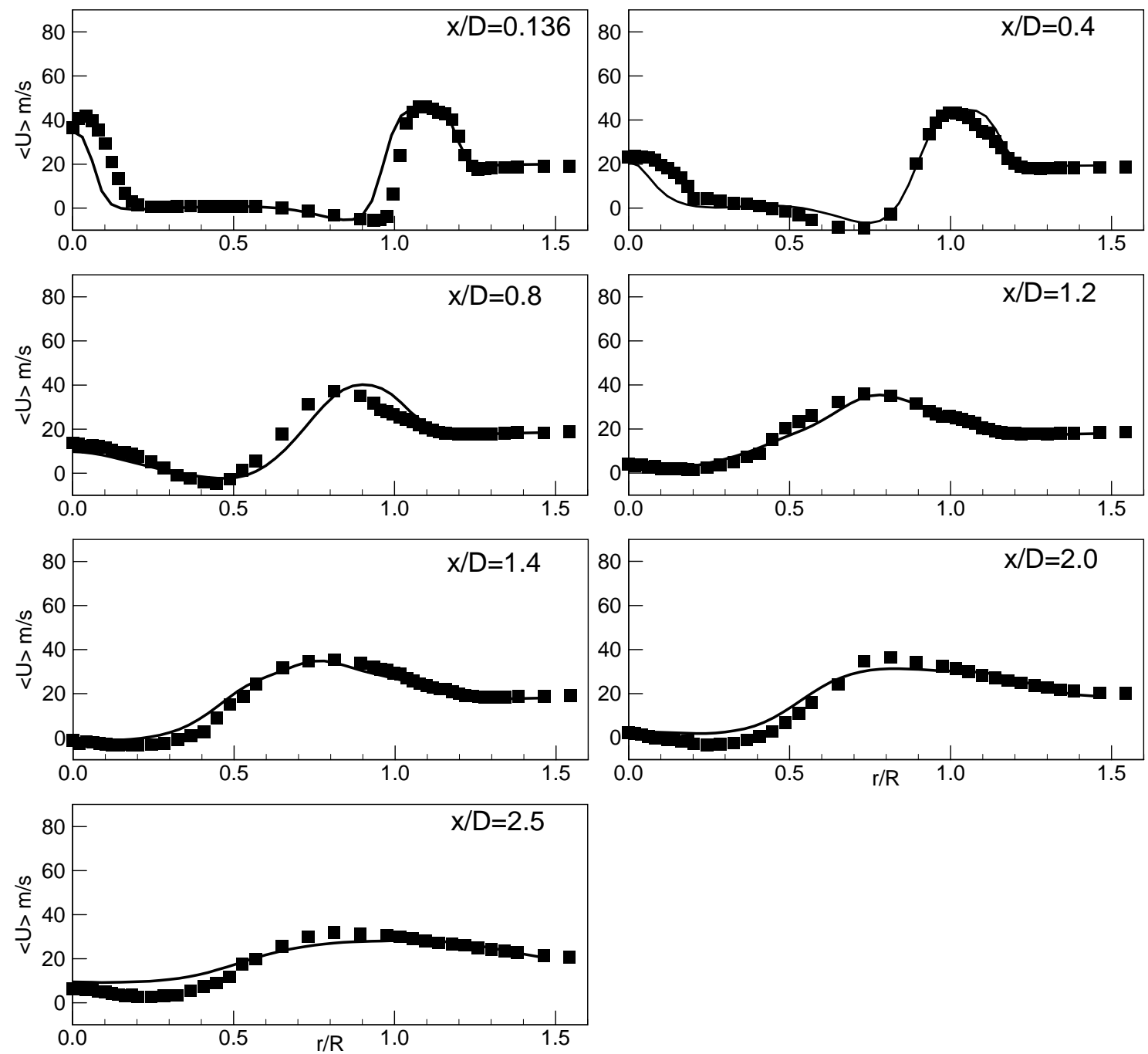

Figure 7: Comparison of mean axial velocity. Lines represent LES results, and symbols represent experimental measurements. 

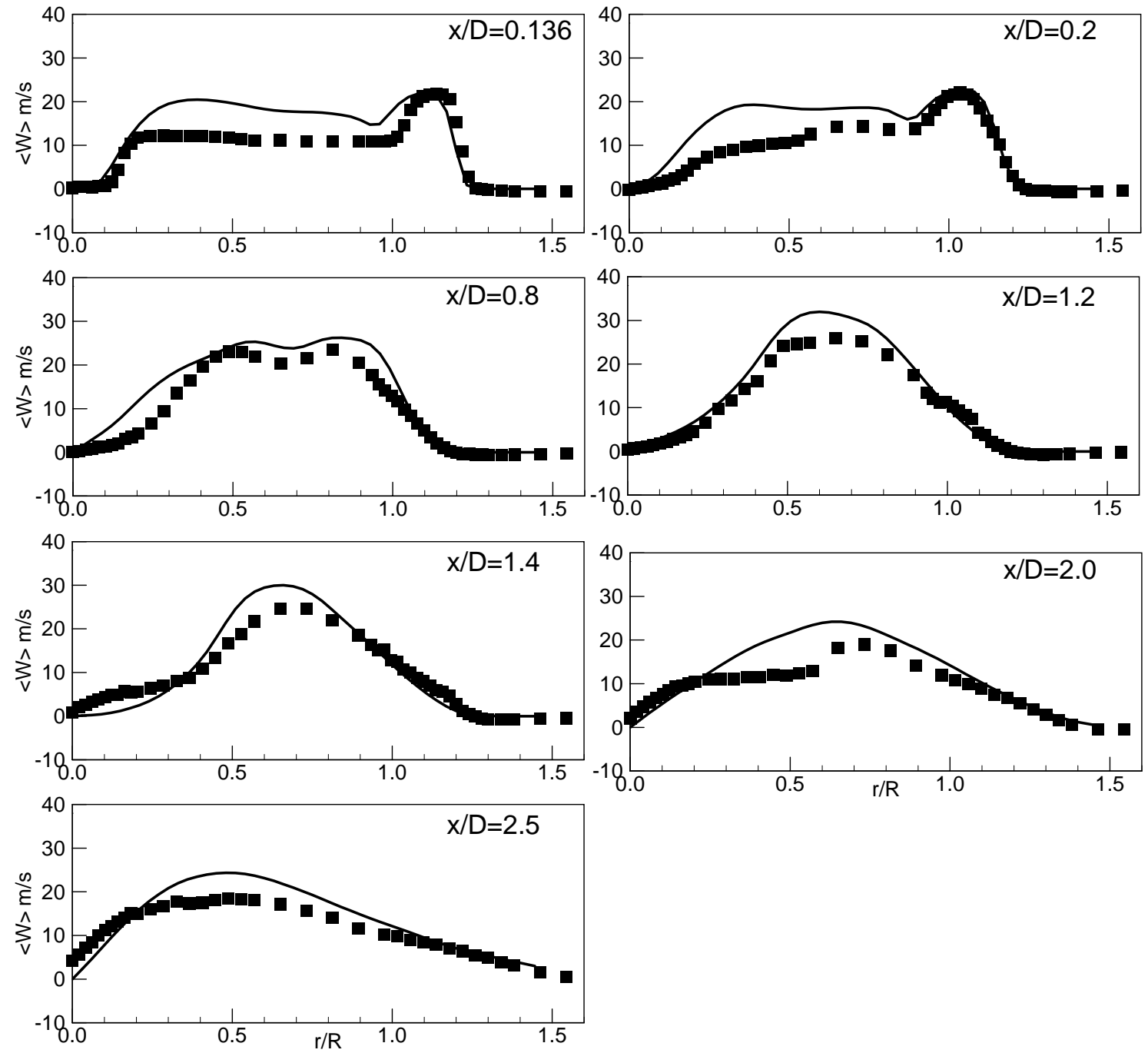

Figure 8: Comparison of mean swirling velocity, Lines represent LES results, and symbols represent experimental measurements. 

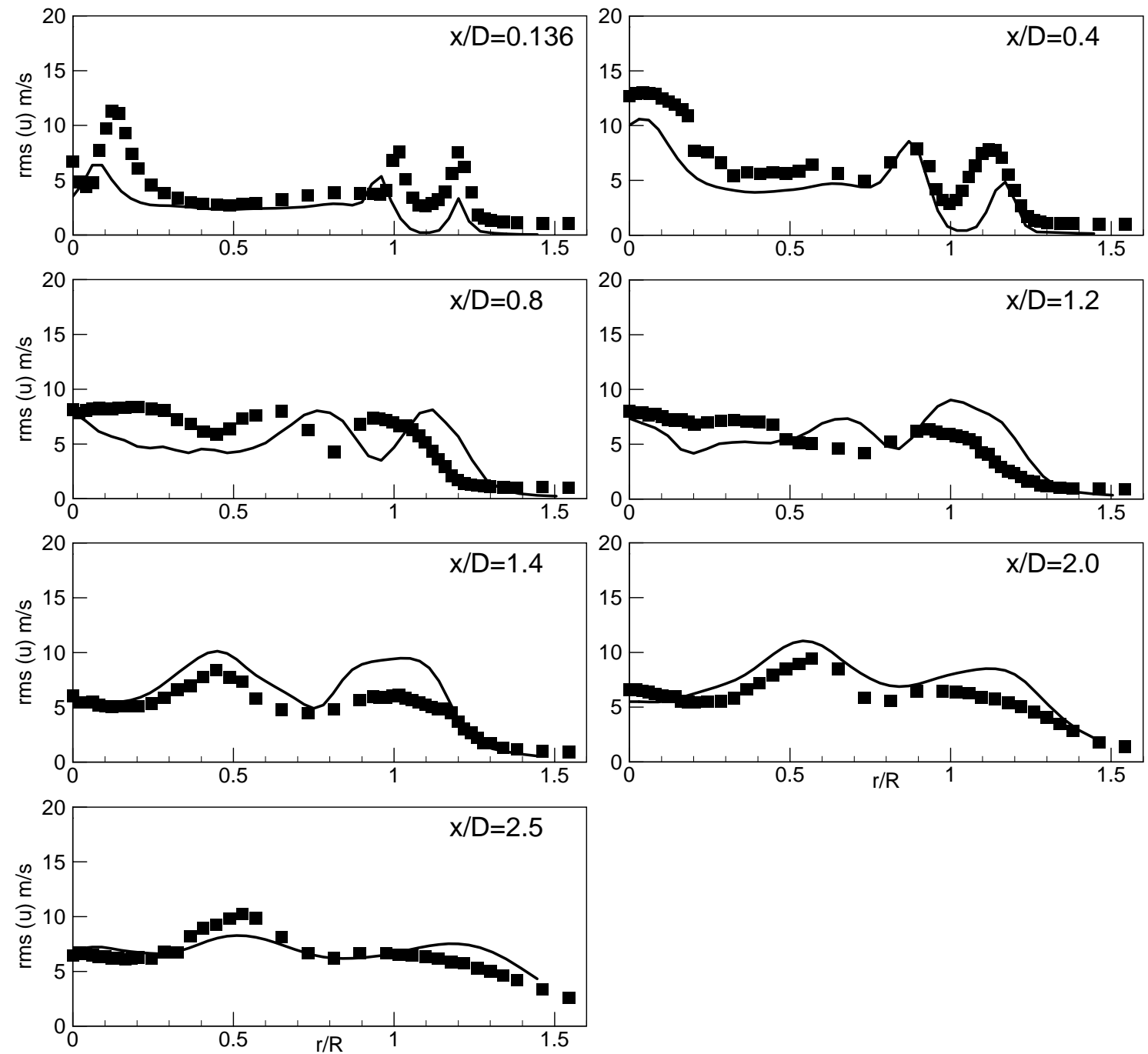

Figure 9: Comparison of rms axial velocity. Lines represent LES results, and symbols represent experimental measurements. 

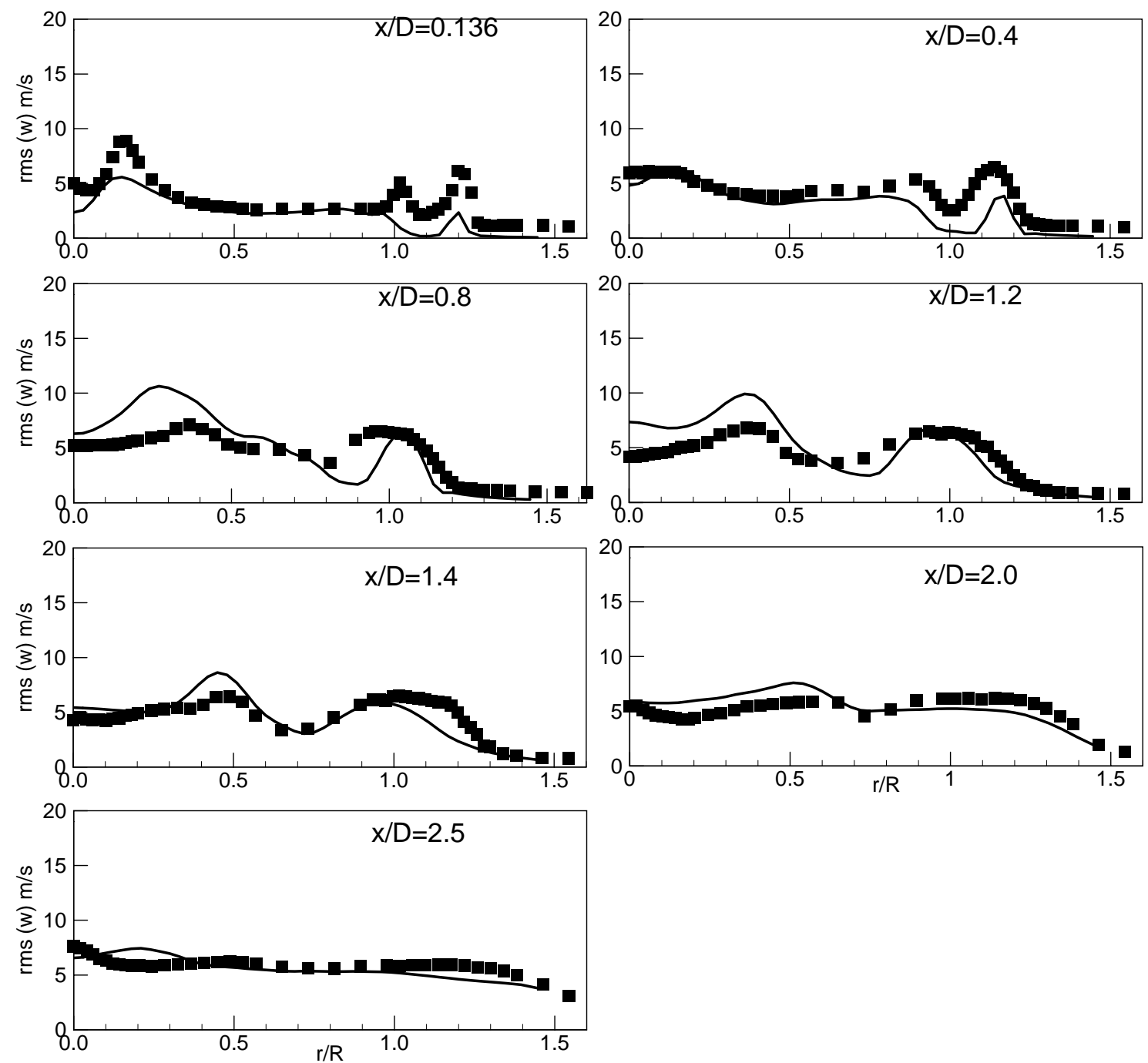

Figure 10: Comparison of rms swirling velocity. Lines represent LES results, and symbols represent experimental measurements. 

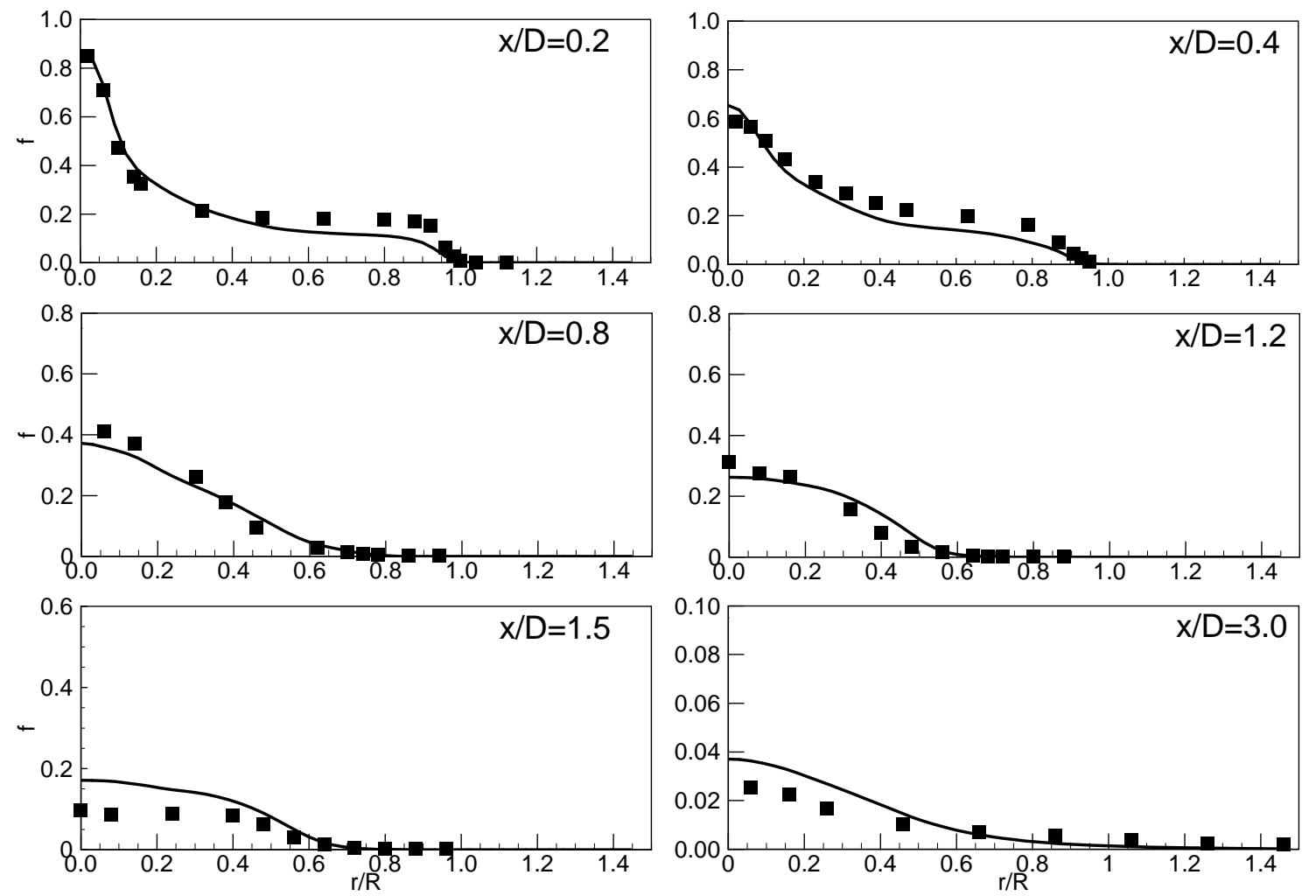

Figure 11: Comparison of mean mixture fraction. Lines represent LES results, and symbols represent experimental measurements. 

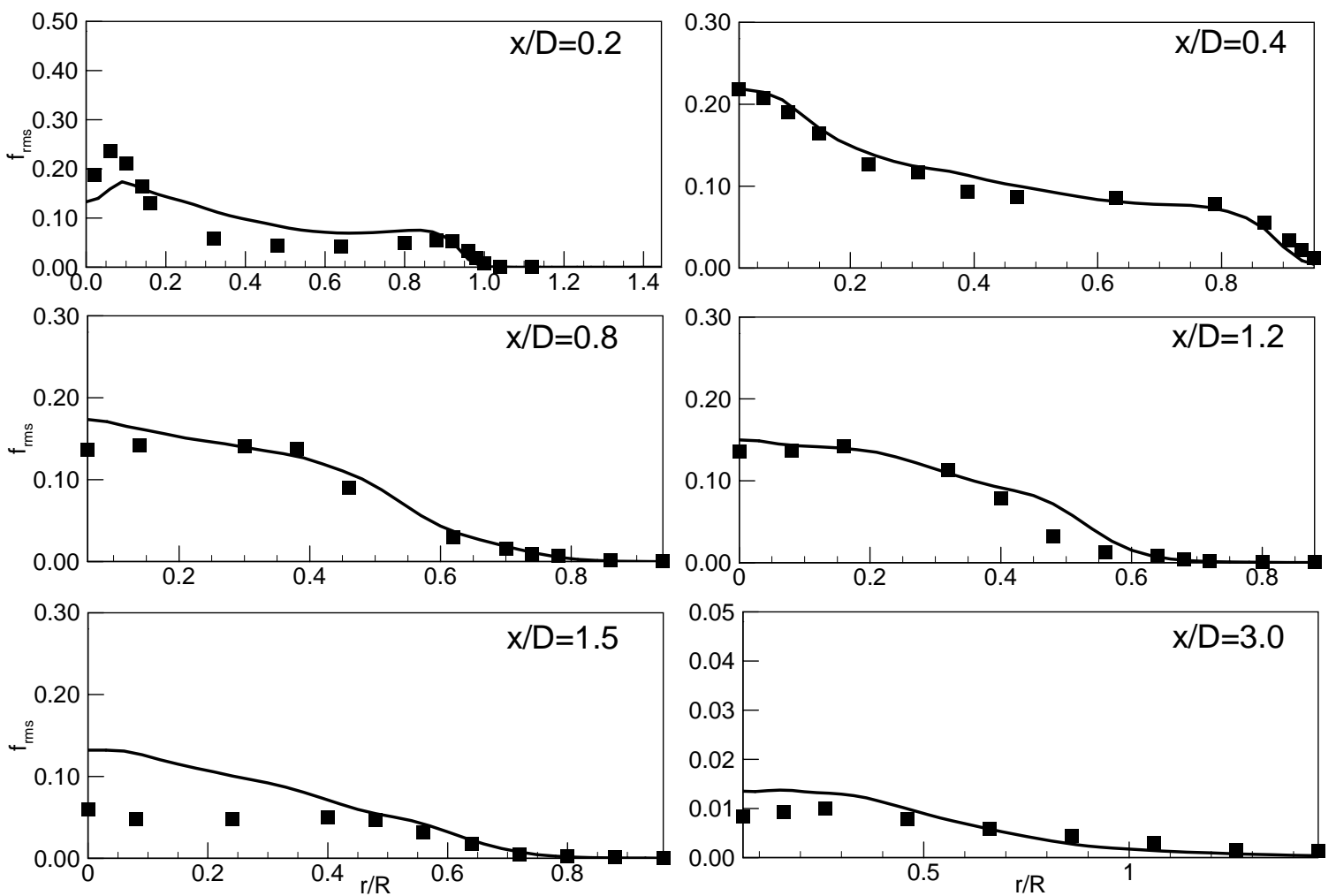

Figure 12: Comparison of mixture fraction variance. Lines represent LES results, and symbols represent experimental measurements. 

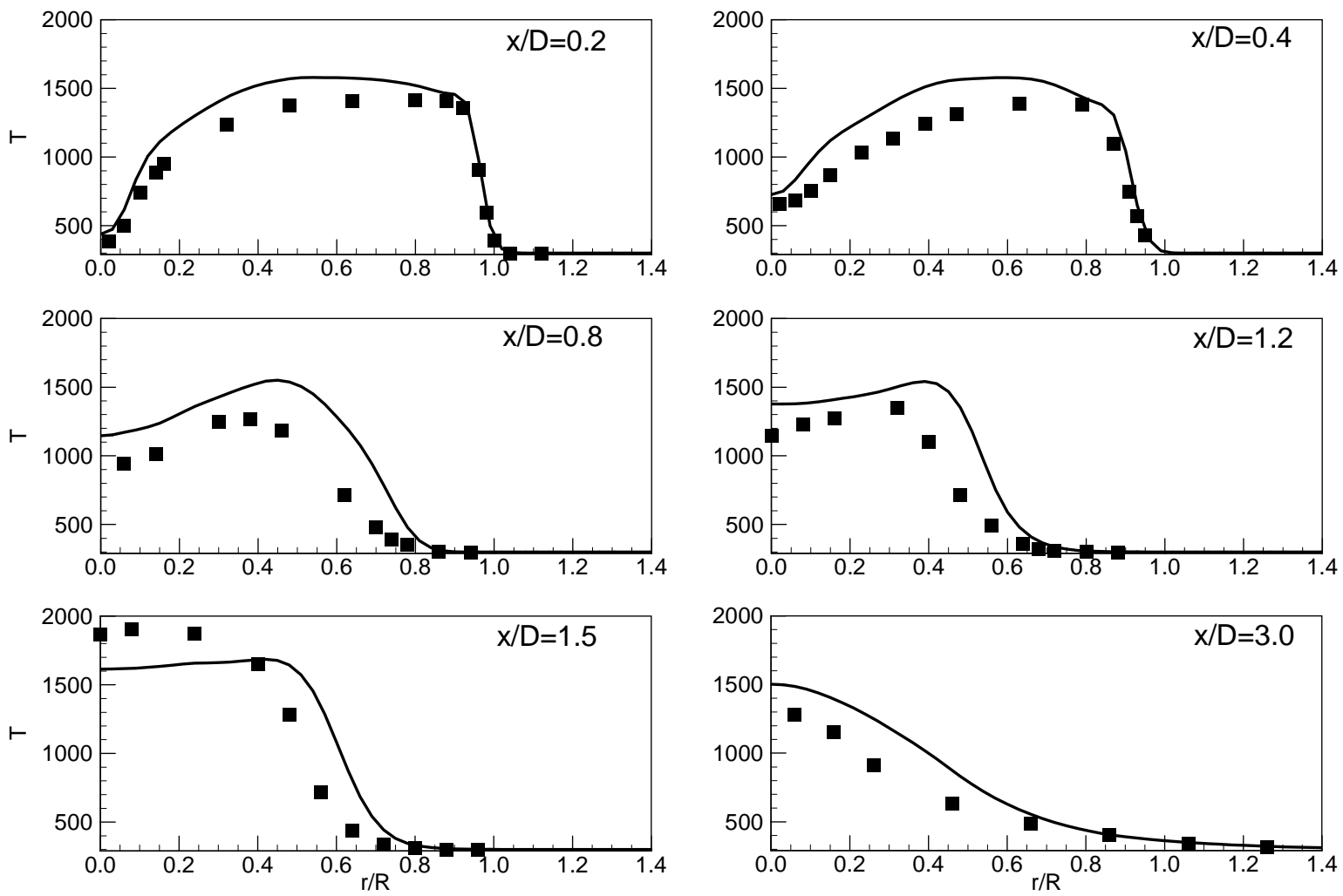

Figure 13: Comparison of mean temperature. Lines represent LES results, and symbols represent experimental measurements. 

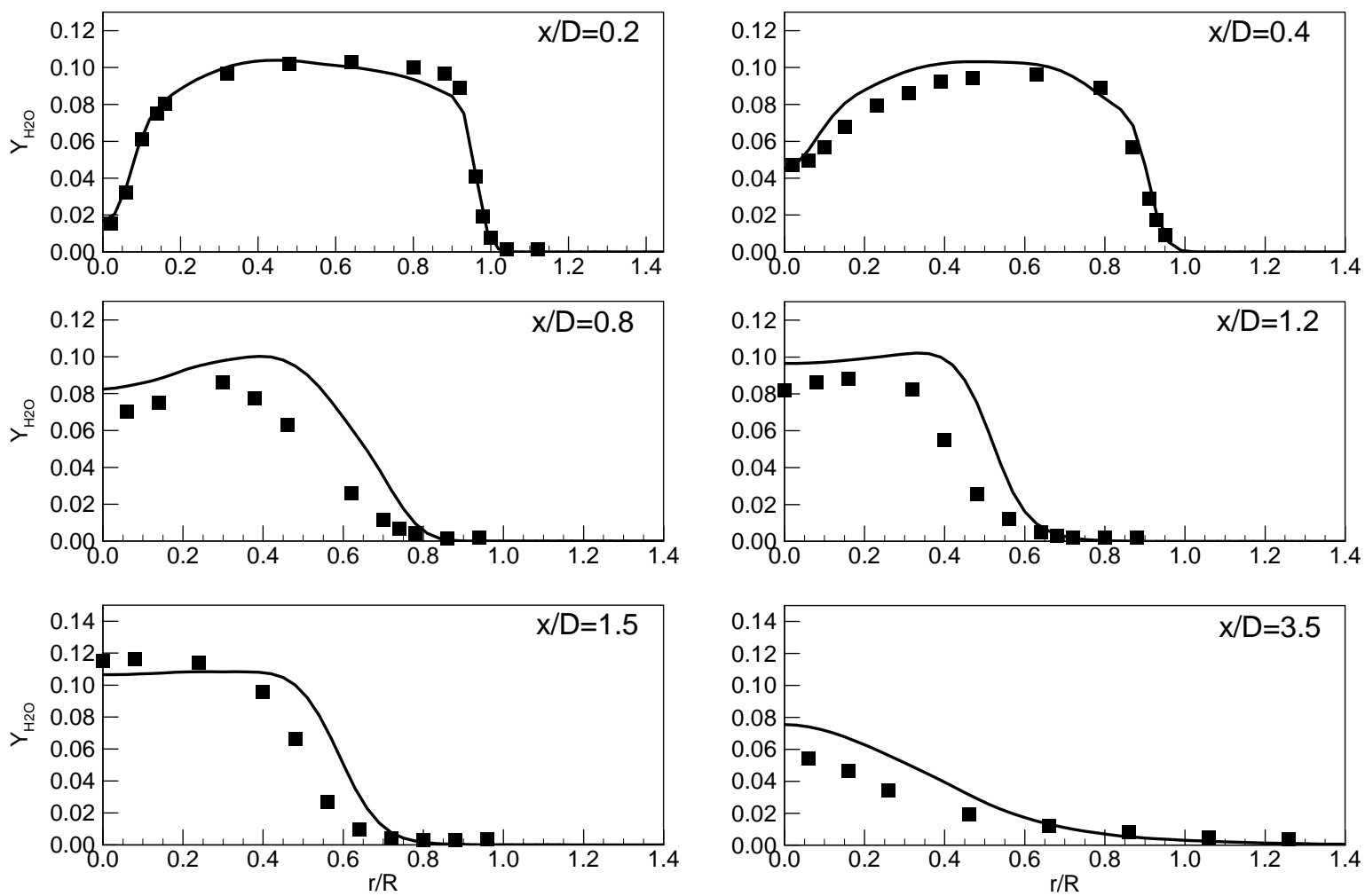

Figure 14: Comparison of mass fraction of $\mathrm{H}_{2} \mathrm{O}$. Lines represent LES results, and symbols represent experimental measurements. 

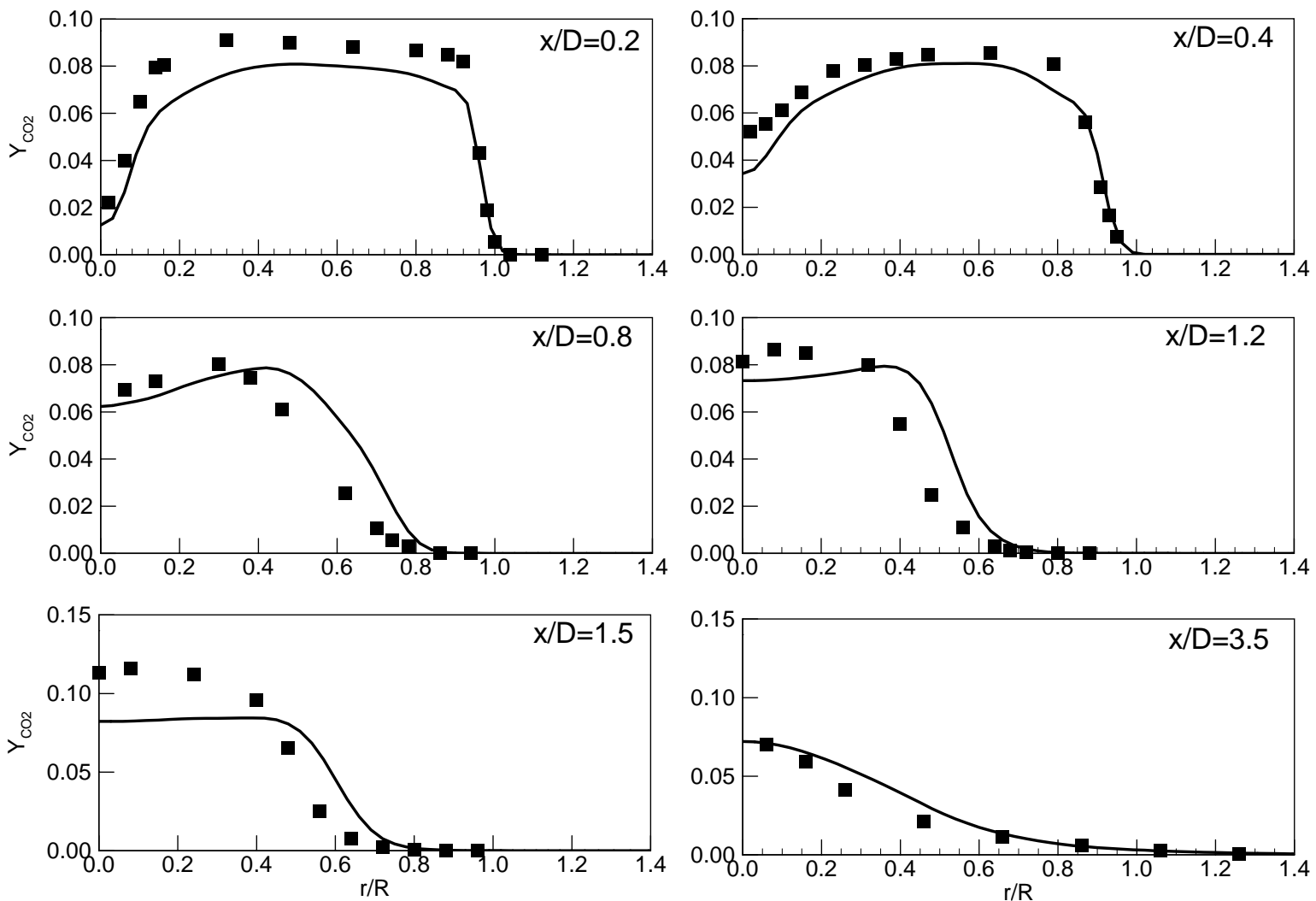

Figure 15: Comparison of mass fraction of $\mathrm{CO}_{2}$. Lines represent LES results, and symbols represent experimental measurements. 

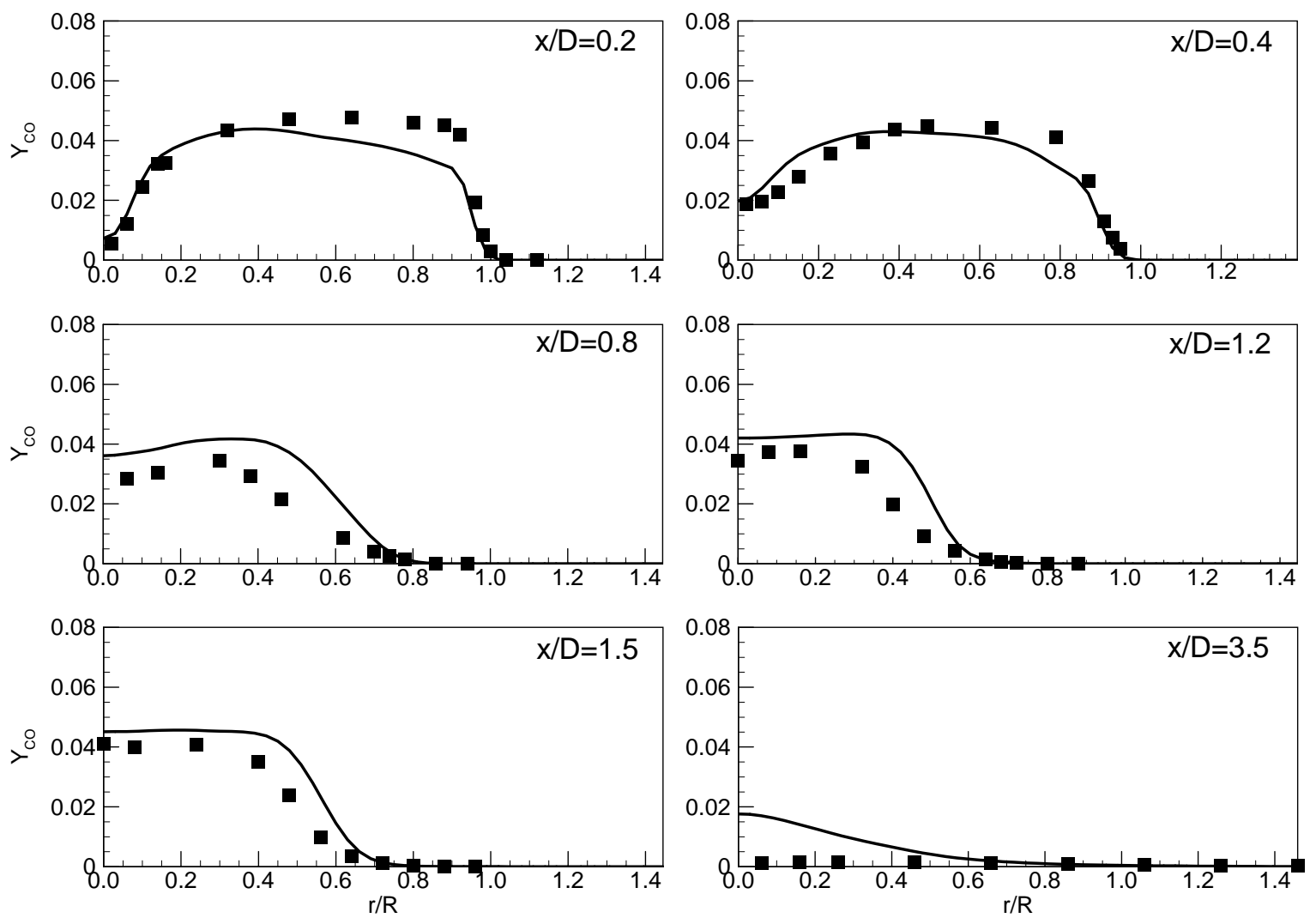

Figure 16: Comparison of mass fraction of $C O$. Lines represent LES results, and symbols represent experimental measurements. 